\title{
The Riesz transform for homogeneous Schrödinger operators on metric cones
}

\author{
Andrew Hassell and Peijie Lin
}

\begin{abstract}
We consider Schrödinger operators on a metric cone whose cross section is a closed Riemannian manifold $(Y, h)$ of dimension $d-1 \geq 2$. Thus the metric on the cone $M=(0, \infty)_{r} \times Y$ is $d r^{2}+r^{2} h$. Let $\Delta$ be the Friedrichs Laplacian on $M$ and let $V_{0}$ be a smooth function on $Y$ such that $\Delta_{Y}+V_{0}+(d-2)^{2} / 4$ is a strictly positive operator on $L^{2}(Y)$ with lowest eigenvalue $\mu_{0}^{2}$ and second lowest eigenvalue $\mu_{1}^{2}$, with $\mu_{0}, \mu_{1}>0$. The operator we consider is $H=\Delta+V_{0} / r^{2}$, a Schrödinger operator with inverse square potential on $M$; notice that $H$ is homogeneous of degree -2 .

We study the Riesz transform $T=\nabla H^{-1 / 2}$ and determine the precise range of $p$ for which $T$ is bounded on $L^{p}(M)$. This is achieved by making a precise analysis of the operator $(H+1)^{-1}$ and determining the complete asymptotics of its integral kernel. We prove that if $V$ is not identically zero, then the range of $p$ for $L^{p}$ boundedness is
\end{abstract}

$$
\left(\frac{d}{\min \left(1+d / 2+\mu_{0}, d\right)}, \frac{d}{\max \left(d / 2-\mu_{0}, 0\right)}\right),
$$

while if $V$ is identically zero, then the range is

$$
\left(1, \frac{d}{\max \left(d / 2-\mu_{1}, 0\right)}\right) .
$$

The result in the case of an identically zero $V$ was first obtained in a paper by H.-Q. Li [33].

\section{Introduction}

The Riesz transform $T$ on the Euclidean space $\mathbb{R}^{d}$ is defined by

$$
T=\nabla \Delta_{\mathbb{R}^{d}}^{-1 / 2}
$$

where $\Delta_{\mathbb{R}^{d}}$ is the (positive) Laplacian operator. In this paper we study the Riesz transform $T$ in the more general setting of metric cones.

Mathematics Subject Classification (2010): Primary 42B37; Secondary 58J05, 35J05.

Keywords: Metric cone, Schrödinger operator, Riesz transform, inverse square potential, resolvent. 
A metric cone $M$ is of the form $M=Y \times(0, \infty)$, where $(Y, h)$ is a compact Riemannian manifold with dimension $d-1$. The cone $M$ is equipped with the conic metric $g=d r^{2}+r^{2} h$. The Euclidean space $\mathbb{R}^{d}$ provides the simplest example of a metric cone, with cross section $Y=\mathbb{S}^{d-1}$ with its standard metric. General metric cones enjoy a dilation symmetry analogous to that of Euclidean space, but no other symmetries in general.

The Laplacian on the cone expressed in polar coordinates is

$$
\Delta=-\partial_{r}^{2}-\frac{d-1}{r} \partial_{r}+\frac{1}{r^{2}} \Delta_{Y}
$$

where $\Delta_{Y}$ is the Laplacian on the compact Riemannian manifold $Y$. This operator is positive and symmetric on the domain $C_{c}^{\infty}(Y \times(0, \infty))$, that is on smooth functions supported away from the cone tip. The operator $\Delta$ is defined to be the Friedrichs extension of this symmetric operator. The Riesz transform $T$ on the cone $M$ is then defined by

$$
T=\nabla \Delta^{-1 / 2}
$$

Here $\nabla$ is shorthand for $\left(\partial_{r}, r^{-1} \nabla_{Y}\right)$, or, in other words, we measure the gradient on the cone using the metric $g$. The question of the boundedness of the Riesz transform on cones, i.e., for what $p$ the operator $T$ is bounded on $L^{p}(M)$, was answered by H.-Q. Li in [33]. The characterisation of the boundedness, stated in Theorem 1.1, is in terms of the second smallest eigenvalue of an operator involving $\Delta_{Y}$. We provide a different proof to this result in Section 5 of this paper.

Theorem 1.1. Let $d \geq 3$ and let $M$ be a metric cone with dimension $d$ and cross section $Y$. The Riesz transform $T=\nabla \Delta^{-1 / 2}$ is bounded on $L^{p}(M)$ if and only if $p$ is in the interval

$$
\left(1, \frac{d}{\max \left(d / 2-\mu_{1}, 0\right)}\right),
$$

where $\mu_{1}>0$ is the square root of the second smallest eigenvalue of the operator $\Delta_{Y}+((d-2) / 2)^{2}$.

More significantly, the methods used in this paper to prove Theorem 1.1 can be applied to study the boundedness properties of a more general class of operators, obtained by adding an inverse square potential to the Laplacian. Let $V_{0}: Y \rightarrow \mathbb{C}$ be a smooth function on $Y$ satisfying the condition

$$
\Delta_{Y}+V_{0}(y)+\left(\frac{d-2}{2}\right)^{2}>0
$$

in the strict sense that the bottom of the spectrum of the operator in (1.3) is strictly positive, and define

$$
H=\Delta+\frac{V_{0}}{r^{2}}
$$


Notice that $H$ is homogeneous of degree -2 , like the Laplacian. Condition (1.3) ensures that $H$ is a positive operator (see Proposition 4.1), so $H^{-1 / 2}$ is well defined. We can then define the Riesz transform $T=T_{V_{0}}$ of the Schrödinger operator $H$ by

$$
T=\nabla H^{-1 / 2}=\nabla\left(\Delta+\frac{V_{0}(y)}{r^{2}}\right)^{-1 / 2} .
$$

Notice that (1.3) allows our potential $V=V_{0} / r^{2}$ to be "a bit negative"; in particular, it allows $V_{0}$ to be any constant greater than $-(d-2)^{2} / 4$.

The goal of this article is to find the exact interval for $p$ on which the Riesz transform $T$ with an inverse square potential $V=V_{0} / r^{2}$ is bounded on $L^{p}(M)$, where $M$ is a metric cone with dimension $d \geq 3$.

A necessary condition, stated in Theorem 1.2, for the boundedness was found in [27] by C. Guillarmou and the first author, in the slightly different setting of asymptotically conic manifolds. These are complete Riemannian manifolds $\left(M^{\circ}, g\right)$ such that $M^{\circ}$ is the interior of a compact manifold $\bar{M}$ with boundary, and $g$ has a prescribed singularity at the boundary of $\bar{M}$. Precisely, there exists a boundary defining function $x$ for $\bar{M}$, and a family of metrics $h(x)$ on $\partial \bar{M}$, such that the metric $g$ has the form

$$
\frac{d x^{2}}{x^{4}}+\frac{h(x)}{x^{2}}
$$

in a collar neighbourhood of $\partial \bar{M}$. Here $r=1 / x$ behaves like the radial coordinate on the cone over $\partial \bar{M}$; the metric in terms of $r$ reads $g=d r^{2}+r^{2} h(1 / r)$, so is asymptotic to the conic metric $d r^{2}+r^{2} h(0)$ as $r \rightarrow \infty$. In [27], potentials of the form $V \in x^{2} C^{\infty}(\bar{M})$ were considered; that is, the potentials decay as $r^{-2}$ at infinity, and the limiting 'potential at infinity' $V_{0}$ was defined by $V_{0}:=\left.x^{-2} V\right|_{\partial \bar{M}}$.

Theorem 1.2 (Theorem 1.5 in [27]). Let $d \geq 3$ and let $\left(M^{\circ}, g\right)$ be an asymptotically conic manifold with dimension $d$. Consider the operator $\mathbf{P}=\Delta_{g}+V$ with $V \in x^{2} C^{\infty}(\bar{M})$ satisfying

$$
\Delta_{\partial M}+V_{0}+\left(\frac{d-2}{2}\right)^{2}>0 \quad \text { on } L^{2}(Y), \quad \text { where } V_{0}=\left.\frac{V}{x^{2}}\right|_{\partial M} .
$$

Let $\mu_{0}>0$ be the square root of the lowest eigenvalue of the operator (1.6). Suppose that $\mathbf{P}$ has neither zero modes nor zero resonances ${ }^{1}$ and that $V_{0} \not \equiv 0$. Then $\nabla \mathbf{P}^{-1 / 2}$ is unbounded on $L^{p}(M)$ if $p$ is outside the interval

$$
\left(\frac{d}{\min \left(d / 2+1+\mu_{0}, d\right)}, \frac{d}{\max \left(d / 2-\mu_{0}, 0\right)}\right) .
$$

The counterexample used in [27] to show the unboundedness of the Riesz transform can be easily adapted to the context of metric cones, so a similar result also holds for metric cones. Therefore the task now is to find a sufficient condition

\footnotetext{
${ }^{1} \mathrm{~A}$ zero mode, resp. zero resonance, for $P$ is a solution $u$ to the equation $P u=0$ such that $u \in L^{2}\left(M^{\circ}\right)$, resp. $u=O\left(r^{-(d-2)}\right)$, as $r \rightarrow \infty$, but $u \notin L^{2}\left(M^{\circ}\right)$. Zero resonances only occur for $d \leq 4$.
} 
for boundedness. We will see that the sufficient condition involves the same interval (1.7) as in Theorem 1.2, so this interval gives us a complete characterisation of the boundedness of $T$ when $V \not \equiv 0$. Our main result is as follows.

Theorem 1.3. Let $d \geq 3$ and let $M$ be a metric cone with dimension $d$ and cross section $Y$. Let $V_{0}$ be a smooth function on $Y$ that satisfies (1.3). The Riesz transform $T$ with the inverse square potential $V=V_{0} / r^{2}$ is bounded on $L^{p}(M)$ for $p$ in the interval

$$
\left(\frac{d}{\min \left(1+d / 2+\mu_{0}, d\right)}, \frac{d}{\max \left(d / 2-\mu_{0}, 0\right)}\right)
$$

where $\mu_{0}>0$ is the square root of the smallest eigenvalue of the operator $\Delta_{Y}+$ $V_{0}(y)+((d-2) / 2)^{2}$.

Moreover, for any $V \not \equiv 0$, the interval (1.8) characterises the boundedness of $T$, i.e., $T$ is bounded on $L^{p}(M)$ if and only if $p$ is in the interval (1.8).

Remark 1.4. If we specialize to positive potentials, i.e., $V \geq 0$ and $V \not \equiv 0$, then $\mu_{0}>(d-2) / 2$, and we see that the lower threshold for $L^{p}$ boundedness is 1 , and the upper threshold is always greater than $d$. On the other hand, for negative potentials $V$, i.e., $V \leq 0$ and $V \not \equiv 0$, the lower threshold for $L^{p}$ boundedness is always greater than 1 and strictly less than 2, while the upper threshold is strictly less than $d$ but strictly larger than 2 .

Remark 1.5. Some of these results are implied by recent papers of Assaad [4] and Assaad-Ouhabaz [5] dealing with more general classes of potentials on $\mathbb{R}^{d}$ or on complete Riemannian manifolds; see the end of Section 1.2 for further discussion.

An immediate application of Theorem 1.3 is to show that the converse of the second part of Theorem 1.5 in [27], i.e., Theorem 1.2, is also true. As noted in Remark 1.7 of [27], Theorem 1.3 is exactly the missing ingredient. Therefore we have the following result.

Theorem 1.6. Let $\left(M^{\circ}, g\right), \mathbf{P}$, and $\mu_{0}$ be as in Theorem 1.2. Then the Riesz transform $\nabla P^{-1 / 2}$ is bounded on $L^{p}(M)$ if and only if $p$ is in the interval (1.7).

A special case of Theorem 1.3 is the following result on the Riesz transforms with constant non-zero $V_{0}$, in which the boundedness interval is written in terms of the constant.

Corollary 1.7. Let $M$ be a metric cone with dimension $d \geq 3$ and cross section $Y$. The Riesz transform $T=\nabla\left(\Delta+c / r^{2}\right)^{-1 / 2}$, where $c>-((d-2) / 2)^{2}$ and $c \neq 0$, is bounded on $L^{p}(M)$ if and only if $p$ is in the interval

$$
\left(\frac{2 d}{\min \left(d+2+\sqrt{(d-2)^{2}+4 c}, 2 d\right)}, \frac{2 d}{\max \left(d-\sqrt{(d-2)^{2}+4 c}, 0\right)}\right) .
$$




\subsection{Strategy of the proof}

Using functional calculus, we get the expression

$$
T=\frac{2}{\pi} \int_{0}^{\infty} \nabla\left(H+\lambda^{2}\right)^{-1} d \lambda .
$$

We then exploit the homogeneity (of degree -2) of $H$ under dilation of the cone. Thanks to this homogeneity, we obtain the resolvent kernel for $\left(H+\lambda^{2}\right)^{-1}$ from $(H+1)^{-1}$ by scaling the variables; see (5.2). So it suffices to analyze $P:=(H+1)^{-1}$. We do this on a compactified and blown-up space, which is designed so that the asymptotics of its kernel in different regimes can be understood. We use $y$ as a local coordinate on the cross section $Y$. We particularly want to distinguish the diagonal behaviour of the kernel $P^{-1}\left(r, y, r^{\prime}, y^{\prime}\right)$, from the behaviour as $r$ or $r^{\prime}$ tends to zero or infinity. If we consider the kernel as living on $(Y \times[0, \infty])^{2}$, as in Figure 1, then this has the defect that the diagonal meets the boundary hypersurfaces $\{r=0\}$, $\left\{r^{\prime}=0\right\},\{r=\infty\}$, and $\left\{r^{\prime}=\infty\right\}$, making the different asymptotic behaviours difficult to distinguish. To remedy this we perform blowups, as in [27]. As noted in that paper, the operator $r P r$ is elliptic as a $b$-differential operator near $r=0$, that is, an elliptic combination of the ' $b$-vector fields' $r \partial_{r}$ and $\partial_{y_{i}}$. On the other hand, as $r \rightarrow \infty, P$ is an elliptic scattering differential operator, which is to say that it has an expression that looks like the Euclidean Laplacian in polar coordinates as $r \rightarrow \infty$, being an elliptic combination of $\partial_{r}$ and $r^{-1} \partial_{y_{i}}$. Correspondingly we perform the $b$-blowup (used to define the $b$-calculus; see Section 2.1) for small $r$, that is, we blow up the corner $r=r^{\prime}=0$. On the other hand, for large $r$ we perform two blowups (used to define the scattering calculus; see Section 2.2), namely we first blow up the corner $r=r^{\prime}=\infty$, followed by the boundary of the lifted diagonal at $r=\infty$, obtaining the space $M_{b, \mathrm{sc}}^{2}$ illustrated in Figure 1 . Now the diagonal is separated from the boundary hypersurfaces in Figure 1 and on this blown-up space, we can more easily construct the kernel of $P^{-1}$ and describe the different types of asymptotics.
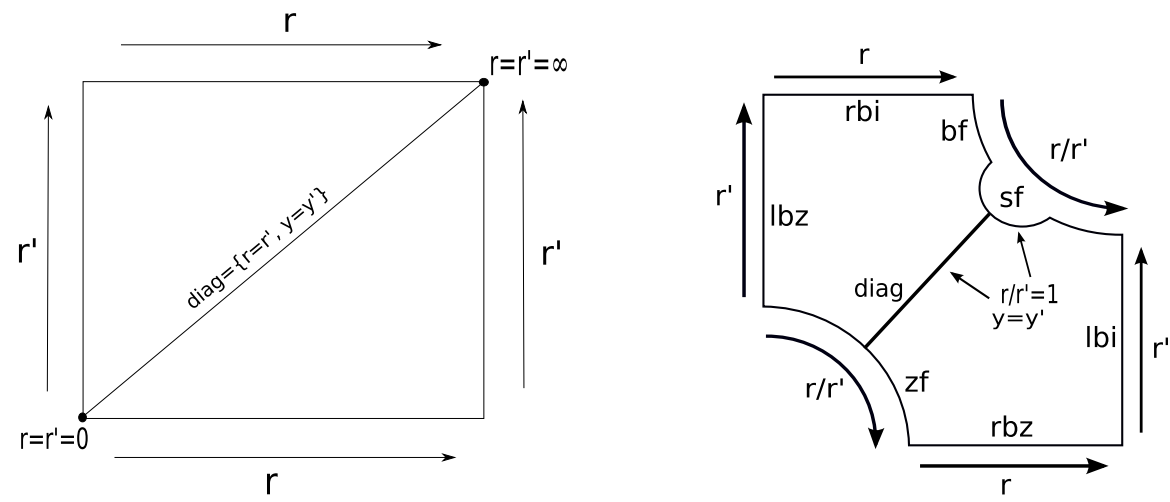

Figure 1 . The space $(Y \times[0, \infty])^{2}$, left, and the blown-up double space $M_{b, \mathrm{sc}}^{2}$ after three blowups, right. 
Because the kernel behaves differently in different parts of the blown-up space, and especially because we use different calculi near the two hypersurfaces zf and sf, we break the blown-up space into different regions, and construct the resolvent kernel in each region separately using different tools and techniques. In the end we patch together the constructions in these different regions to obtain the overall resolvent kernel. This construction of the resolvent kernel of $H$, i.e., the kernel of $P^{-1}$, is made in Section 4.

In Section 5, equipped with the knowledge of the behaviours of the kernel of $P^{-1}$ at different parts of the blown-up space, we determine the boundedness properties of the Riesz transform $T$. Using a smooth partition of unity on the blown-up space, we calculate the integral (1.10) and then break the kernel of $T$ up into a near-diagonal part and an off-diagonal part. The near-diagonal part is a Calderón-Zygmund kernel and is bounded on $L^{p}$ for all $p \in(1, \infty)$, while the off-diagonal part is bounded on a typically smaller range of $p$ determined by the leading asymptotic behaviours at the boundary hypersurfaces marked 'lbz' or 'rbz' in Figure 1.

\subsection{Relation to previous work}

Cones have been studied since the 19th century. In particular, the problem of wave diffraction from a cone point, which is important in applied mathematics, was studied by A. Sommerfeld in the 1890s [45]. Other notable early papers include [23] and [24] by F. G. Friedlander and [9] by A. Blank and J. B. Keller. Functional calculus for Laplacians defined on cones was studied by J. Cheeger and M. Taylor in [15] and [16]. Many papers have been written about spaces with cone-like singularities. For example, the Laplacian and heat kernel on compact Riemannian manifolds with cone-like singularities were studied in [17] by J. Cheeger and in [35] by E. Mooers. In [11], J. Brüning and R. Seeley studied the Laplacian on manifolds with an asymptotically conic singularity, and in [36] R. B. Melrose and J. Wunsch studied the wave equation and diffraction on spaces with asymptotically conic singularities.

The classical case of the Riesz transform on the Euclidean space $\mathbb{R}^{d}$ goes back to the 1920s, and the case of one dimension (the Hilbert transform) was studied by M. Riesz in [40]. The paper [44] by R. S. Strichartz is the first paper that studies the Riesz transform on a complete Riemannian manifold. In [18], T. Coulhon and X. T. Duong proved that the Riesz transform on a complete Riemannian manifold, satisfying the doubling condition and the diagonal bound on the heat kernel, is of weak type $(1,1)$, and hence is bounded on $L^{p}$ for $1<p \leq 2$. Since then there have been many studies of the Riesz transform. For example, the Riesz transform on complete Riemannian manifolds has been studied in [19], [7], [8], and [20]. Riesz transforms on Lie groups are analyzed in [2], [3], [21], and [41], and Riesz transforms on second order elliptic operators are considered in [10] and [22].

Many papers have been written on Schrödinger operators with an inverse square potential. We only mention a few of the most relevant ones here. In [46], X. P. Wang studied the perturbations of such operators. In [13], G. Carron studied Schrödinger operators with potentials that are homogeneous of degree -2 near 
infinity. In [12], N. Burq, F. Planchon, J. G. Stalker, and A. S. Tahvildar-Zadeh generalise the standard Strichartz estimates for the Schrödinger equation and the wave equation to the case in which an additional inverse square potential is present. In [30] the first author and A. Sikora investigated one-dimensional Riesz transforms, including with inverse square potentials, with respect to measures of the form $r^{d-1} d r$, thus mimicking the measure on a $d$-dimensional cone.

Now we turn to past results on the boundedness of the Riesz transform $T$ with a potential $V$ on metric cones. We have already mentioned the result (Theorem 1.1) of H.-Q. Li for $V \equiv 0$, and the work [27] of C. Guillarmou and the first author on asymptotically conic manifolds. The method from [27] was based in part on the paper [14]. In [28] the two authors performed a similar analysis but allowed certain zero modes and zero resonances. In [6], P. Auscher and B. Ben Ali obtained a result on $\mathbb{R}^{d}$, stated in Theorem 1.8, which involves the reverse Hölder condition. It is an improvement of the earlier results by Z. W. Shen in [42].

Theorem 1.8 (Theorem 1.1 in [6]). Let $1<q \leq \infty$. If $V$ is in the reverse Hölder class $B_{q}$, then for some $\varepsilon>0$ depending only on $V$ the Riesz transform with potential $V$ is bounded on $L^{p}\left(\mathbb{R}^{d}\right)$ for $1<p<q+\varepsilon$.

The reverse Hölder condition $V \in B_{q}$ implies that $V>0$ almost everywhere and $V \in L_{\text {loc }}^{q}\left(\mathbb{R}^{d}\right)$. A positive inverse square potential is in $B_{q}$ if and only if $q<d / 2$. So this theorem gives boundedness for $1<p<d / 2$, which is smaller than the range obtained in Theorem 1.3 for positive inverse square potentials (of course this is a very small subclass of $B_{q}$-potentials).

Very recently, Assaad and Assaad-Ouhabaz have proved results for Riesz transforms of Schrödinger operators which include some of our results. The following result is from [4].

Theorem 1.9. Let $M$ be a complete noncompact Riemannian manifold with dimension $d \geq 3$. Suppose that the function $V \leq 0$ satisfies $\Delta+(1+\varepsilon) V \geq 0$, the Sobolev inequality

$$
\|f\|_{L^{\frac{2 d}{d-2}}(M)} \lesssim\|\nabla f\|_{L^{2}(M)}
$$

holds for all $f \in C_{0}^{\infty}(M)$, and that $M$ is of homogeneous type, i.e., for all $x \in M$ and $r>0$,

$$
\mu(B(x, 2 r)) \lesssim \mu(B(x, r))
$$

where $\mu$ is the measure on $M$. Then the Riesz transform $T=\nabla(\Delta+V)^{-1 / 2}$ is bounded on $L^{p}(M)$ for all $p$ in the interval

$$
\left(\frac{2 d}{d+2+(d-2) \sqrt{\varepsilon /(\varepsilon+1)}}, 2\right] .
$$

Except for the fact that they are not necessarily smooth manifolds in a neighbourhood of the cone tip at $r=0$, metric cones satisfy the conditions of Theorem 1.9. So this result can be directly compared with ours in the case of Schrödinger 
operators of the form $\left(\Delta+c / r^{2}\right)$, where the constant $c$ satisfies $-((d-2) / 2)^{2}<c<0$. In that case the lower threshold in (1.9) given by Corollary 1.7 is the same as the lower threshold in (1.12) given by J. Assaad's result. Also in [4] it is shown that the Riesz transform for Schrödinger operators with a signed potential in $L^{d / 2}$, or a nonnegative potential in $L^{d / 2, \infty}$, on a $d$-dimensional Riemannian manifold obeying the Sobolev inequality (1.11) are bounded on $(1, d)$ provided that this is true for the Riesz transform with zero potential. Note that this case just fails to cover negative inverse square potentials on cones, which are in $L^{d / 2, \infty}$. Further results on signed potentials are proved by J. Assaad and E. Ouhabaz in [5].

\section{Review of the $b$-calculus and the scattering calculus}

In this section we briefly recall the key elements of the $b$-calculus and the scattering calculus that we require in Section 4. For more details, see [37] or [26] for the $b$ calculus, and [38], [39] for the scattering calculus.

\section{1. $b$-calculus}

Let $X$ be a manifold with boundary with boundary defining function $x$ (that is, $\partial X=\{x=0\}$ and $d x \neq 0$ on $\partial X)$. A differential operator on $X$ is called a $b$-differential operator if it is generated over $C^{\infty}(X)$ by vector fields tangent to the boundary of $X$. Near $\partial X$ such vector fields are linear combinations of vector fields $x \partial_{x}$ and $\partial_{y_{i}}$ in terms of a local coordinate system $\left(x, y_{1}, \ldots, y_{d-1}\right)$ with $\left(y_{1}, \ldots, y_{d-1}\right)$ restricting to a local coordinate system on $\partial X$.

The $b$-calculus is a "microlocalization" of the set of $b$-differential operators. To define the $b$-calculus, we first blow up ${ }^{2} X^{2}$ along $(\partial X)^{2}$ to obtain the blown-up manifold $X_{b}^{2}=\left[X^{2} ;(\partial X)^{2}\right]$, called the $b$-double space. This produces a manifold with corners which has three boundary hypersurfaces: one defined by $x / x^{\prime}=0$ (here and below we use the convention that unprimed variables on the double space are coordinates on the left copy, and primed variables are coordinates on the right copy), one defined by $x^{\prime} / x=0$, and one defined by $x+x^{\prime}=0$. These are usually denoted lb, rb, and ff, respectively, but, in accordance with our notational conventions for the space in Figure 1, here we will call them lbz, rbz and zf (here the ' $\mathrm{z}$ ' stands for 'zero' and refers to the fact that the $b$-blowup takes place at $\left.r=r^{\prime}=0\right)$.

It is convenient to regard elements of the $b$-calculus as acting on $b$-half densities. We define a smooth $b$-half-density on $X$ to be a smooth half-density in the interior of $X$ taking the form $f\left|d x / x d y_{1} \ldots d y_{d-1}\right|^{1 / 2}$ near the boundary, with $f \in C^{\infty}(X)$. We say that such a $b$-half-density is nonvanishing if it is nonvanishing as a halfdensity in the interior of $X$, and if $f>0$. Let $\kappa$ be a fixed smooth nonvanishing $b$-half-density on $X$ and let $\kappa \otimes \kappa$ denote the tensor product of $\kappa$ in each of the left and right factors of $X$, lifted to $X_{b}^{2}$.

\footnotetext{
${ }^{2}$ Here and below we use 'blow up' to mean real blow up; as a set, the manifold $[X ; S]$ obtained by blowing up $X$ at the submanifold $S$ is obtained by removing $S$ and replacing it with its inward pointing spherical normal bundle. It is endowed with a differentiable structure that makes polar coordinates around $S$ smooth functions on the blown up space.
} 
Definition 2.1 (Melrose [37]). An element of the small b-calculus $\Psi_{b}^{m}(X), m \in \mathbb{R}$, is a distribution $u$ on $X_{b}^{2}$ multiplied by $\kappa \otimes \kappa$, where $u$ satisfies

(i) $u$ is conormal of order $m$, in the sense of Definition 18.2.6 and Theorem 18.2.8 of [32], with respect to $\operatorname{diag}_{b}$, smoothly up to the hypersurface zf;

(ii) $u$ vanishes to infinite order at $\mathrm{lbz}$ and $\mathrm{rbz}$.

Using the Schwartz kernel theorem, we interpret these as operators on (smooth) $b$-half-densities on $X$; elements of the space $\Psi_{b}^{0}(X)$ extend to bounded operators on $L^{2}$ half-densities. We also define

$$
\Psi_{b}^{-\infty}(X)=\bigcap_{m} \Psi_{b}^{m}(X)
$$

such operators are smooth $b$-half-densities on $X_{b}^{2}$ that vanish to infinite order at lbz and rbz.

The $b$-calculus is closed under composition; see Proposition 5.20 in [37] for the proof of the following proposition.

Proposition 2.2. If $X$ is a compact manifold with boundary then

$$
\Psi_{b}^{m}(X) \circ \Psi_{b}^{m^{\prime}}(X) \subset \Psi_{b}^{m+m^{\prime}}(X),
$$

where $m, m^{\prime} \in \mathbb{R}$.

Since our purpose is to invert elliptic $b$-differential operators, it is important to know about parametrix constructions under the small $b$-calculus. Such parametrix constructions are analogous to the standard elliptic parametrix construction in Theorem 18.1.24 of [32].

Proposition 2.3. If $P$ is an elliptic partial b-differential operator of order $k$, then there exists an operator $G \in \Psi_{b}^{-k}(X)$ such that

$$
\text { Id }-P G \in \Psi_{b}^{-\infty}(X), \quad \text { Id }-G P \in \Psi_{b}^{-\infty}(X) .
$$

Moreover, if $G^{\prime} \in \Psi_{b}^{-k}(X)$ also has this property then $G-G^{\prime} \in \Psi_{b}^{-\infty}(X)$.

For the proof, see Section 4.13 of [37]. This inversion property is not good enough for Fredholm theory, as the error terms Id $-P G$ and Id $-G P$ may not be compact. To investigate when an element in the small $b$-calculus is compact, we introduce the indicial operator.

Definition 2.4. Let $A \in \Psi_{b}^{m}(X)$ be a $b$-pseudodifferential operator. The indicial operator $I_{b}(A)$ is defined to be the restriction of the Schwartz kernel of $A$ to zf.

The indicial operator $I_{b}(A)$ can be interpreted as a translation-invariant operator on the cylinder $\partial X \times \mathbb{R}$. As such $I_{b}$ is an algebra homomorphism:

$$
I_{b}(P A)=I_{b}(P) I_{b}(A) \text {. }
$$

The compactness of an operator is linked to its indicial operator.

Proposition 2.5. Suppose that $X$ is a manifold with corners, and $A \in \Psi_{b}^{m}(X)$ with $m<0$. Then $A$ is compact on $L^{2}(X)$ if and only if $I_{b}(A)=0$. 
When inverting an elliptic partial differential operator in the small $b$-calculus, the error term will usually have a nonzero indicial operator, and therefore will not be compact. In order to obtain an error term whose indicial operator vanishes, we have to expand the small $b$-calculus to a bigger calculus, called the full $b$ calculus, in which the Schwartz kernels are permitted to have polyhomogeneous conormal expansions, i.e., expansions in powers and logarithms, at the boundary hypersurfaces lbz, zf, and rbz.

To define polyhomogeneous cornormal functions, we need the notion of an index set. This is a discrete subset $F \subset \mathbb{C} \times \mathbb{N}_{0}$ such that every 'left segment' $F \cap\{(z, p): \operatorname{Re} z<N\}, N \in \mathbb{R}$ is a finite set. Also, it is assumed that $(z, p) \in F$ implies that $(z+1, p) \in F$ and $(z, q) \in F$ for $q \in \mathbb{N}_{0}, q \leq p$.

Given a boundary hypersurface and an index set, we can define polyhomogeneous conormal functions with respect to it. These are functions behaving like sums of products $x^{z}(\log x)^{p}$ of powers and logarithms in one (and hence any) boundary defining function $x$. These definitions follow Sections 5.10 and 5.22 of [37]. We start by defining $L^{\infty}$-based conormal spaces of functions.

Definition 2.6. Let $X$ be a manifold with boundary, with boundary defining function $x$, and let $a$ be a real number. The conormal space $\mathcal{A}^{a}(X)$ consists of those functions $u$ smooth on the interior of $X$ such that, for any finite set $V_{1}, \ldots, V_{l}$ of vector fields on $X$ tangent to the boundary $\partial X$, we have

$$
x^{-a} V_{1} \ldots V_{l} u \in L^{\infty}(X) .
$$

Similarly, if $X$ is a manifold with corners, with boundary hypersurfaces $H_{1}, \ldots, H_{k}$ and boundary defining functions $x_{1}, \ldots, x_{k}$, let $\mathfrak{a}=\left(a_{1}, \ldots, a_{k}\right)$ denote a multiweight for $\partial X$, that is, a real number $a_{j}$ assigned to each $H_{j}$. The conormal space $\mathcal{A}^{\mathfrak{a}}(X)$ consists of those functions $u$ smooth on the interior of $X$ such that, for any finite set $V_{1}, \ldots, V_{l}$ of vector fields on $X$ tangent to each boundary hypersurface $H_{j}$, we have

$$
x_{1}^{-a_{1}} \ldots x_{k}^{-a_{k}} V_{1} \ldots V_{l} u \in L^{\infty}(X) .
$$

We refer to a derivation by a vector field tangent to the boundary of $X$ as a b-derivative.

Definition 2.7. Let $X$ be a manifold with boundary and let $H$ be its boundary. Given an index set $F$, a smooth function $u$ defined on the interior $X^{\circ}$ of $X$ is called polyhomogeneous conormal as it approaches the boundary $H$ with respect to $F$ if, on a collar neighborhood $[0,1)_{x} \times H$ of $H$, one has

$$
u(x, y) \sim \sum_{(z, p) \in F} a_{z, p}(y) x^{z}(\log x)^{p} \quad \text { as } x \rightarrow 0,
$$

with $a_{z, p}$ smooth on $H$. Here, $\sim$ means that for any $N \in \mathbb{N}$, the tail of the series,

$$
u^{\prime}=u-\sum_{(z, p) \in F, \operatorname{Re} z \leq N} a_{z, p}(y) x^{z}(\log x)^{p}
$$

is in $\mathcal{A}^{N}(X)$. In particular it means that $(2.2)$ is $o\left(x^{N}\right)$. We write $\mathcal{A}^{F}(X)$ for the space of functions on $X$ that are polyhomogeneous conormal with index set $F$. 
We also need to define polyhomogeneous conormal functions on a manifold with corners of codimension 2. Since the definition is local, it suffices to consider a manifold with corners with two boundary hypersurfaces.

Definition 2.8. Let $X$ be a manifold with corners with boundary hypersurfaces $H_{1}$ and $H_{2}$ (which may intersect). Given an index family $\left(F_{1}, F_{2}\right)$ for $X$, a smooth function $u$ defined on the interior $X^{\circ}$ of $X$ is called polyhomogeneous conormal (with respect to $F_{1}$ and $F_{2}$ ) as it approaches the boundary of $X$ if for $(i, j)$ equal to $(1,2)$ or $(2,1)$ it has an expansion at $H_{i}$ polyhomogeneous with respect to $F_{i}$ with coefficients in $\mathcal{A}_{\text {phg }}^{F_{j}}\left(H_{i}\right)$ in the following sense: there exists a collar neighbourhood $[0,1)_{x} \times H_{i}$ of $H_{i}$ and functions $a_{z, p} \in \mathcal{A}_{\mathrm{phg}}^{F_{j}}\left(H_{i}\right),(z, p) \in F_{i}$, polyhomogeneous conormal with respect to $F_{j}$ at $\partial H_{i}=H_{i} \cap H_{j}$ such that for all $N \in \mathbb{N}$,

$$
u-\sum_{(z, p) \in F_{i}, \operatorname{Re} z \leq N} a_{z, p}(y) x^{z}(\log x)^{p} \in \mathcal{A}^{\left(N, b_{j}\right)}(X) .
$$

Here $b_{j}$ is a fixed real number chosen smaller than $\inf \left\{z \mid \exists(z, p) \in F_{j}\right\}$ and $\left(N, b_{j}\right)$ is the multiweight that assigns $N$ to $H_{i}$ and $b_{j}$ to $H_{j}$. We write $\mathcal{A}^{F_{1}, F_{2}}(X)$ for the space of functions just defined.

It turns out that, if we know a priori that $u$ is conormal on a manifold with boundary $X$, then it is relatively straightforward to check whether it is polyhomogeneous conormal with a given index set $F$. This is the content of the next proposition.

Proposition 2.9. Let $X$ be a manifold with boundary and a be a real number. Suppose that $u$ is conormal on $X$ with weight a. Let $F$ be an index set. Then $u \in \mathcal{A}^{F}(X)$ if and only if there exists an expansion as in (2.1) for $u$ near $\partial X$ such that, for all $N \in \mathbb{R}$, the tail (2.2) is o( $\left.x^{N}\right)$. (That is, it is only necessary to check the size of the tail itself, not all of its b-derivatives.)

Proof. The "only if" part of the proposition is trivial. To prove the "if" part, let $u \in \mathcal{A}^{a}(X)$, choose $N \in \mathbb{R}$, and let $u_{N}$ be the polyhomogeneous expansion of $u$ involving terms $(z, p)$ with $\operatorname{Re} z \leq N$, as in (2.2). We need to show that the tail (2.2) is in $\mathcal{A}^{N}(X)$. This is trivial for $N<a$, so assume $N \geq a$. Consider estimating a b-derivative, say $x \partial_{x}\left(u-u_{N}\right)$, of the tail. For any $M>N$, this can be written

$$
x \partial_{x}\left(u-u_{M}\right)-x \partial_{x}\left(u_{M}-u_{N}\right) .
$$

The second term has the required estimate since it is a finite polyhomogeneous expansion corresponding to $(z, p) \in F$ with $\operatorname{Re} z>N$. As for the first term, we write using Taylor's formula, for some $\epsilon^{\prime} \in[0, \epsilon]$ (here we are mimicking the proof of Theorem 18.1.4 in [32]),

$$
\begin{aligned}
& \left(u-u_{M}\right)(x+\epsilon x, y)-\left(u-u_{M}\right)(x, y) \\
& \quad=\epsilon x \partial_{x}\left(u-u_{M}\right)(x, y)+\frac{\epsilon^{2} x^{2}}{2} \partial_{x}^{2}\left(u-u_{M}\right)\left(x+\epsilon^{\prime} x, y\right) \\
& \quad \Longrightarrow\left|\epsilon x \partial_{x}\left(u-u_{M}\right)(x, y)-\left(\left(u-u_{M}\right)(x+\epsilon x, y)-\left(u-u_{M}\right)(x, y)\right)\right| \leq \epsilon^{2} x^{a},
\end{aligned}
$$


using the conormality of $u$ of weight $a$ to bound second order b-derivatives of $u$. Hence we get a bound for any $\epsilon$ and $M$ :

$$
\left|x \partial_{x}\left(u-u_{M}\right)(x, y)\right| \leq \epsilon^{-1}\left|\left(u-u_{M}\right)(x+\epsilon x, y)-\left(u-u_{M}\right)(x, y)\right|+\epsilon x^{a} .
$$

We obtain the required estimate by choosing $\epsilon=x^{N+1-a}$ and then $M=2 N+2-a$, as $u-u_{M}=o\left(x^{M}\right)$ by hypothesis. Higher derivatives can be treated iteratively.

A similar result holds for manifolds with corners.

Proposition 2.10. With notation as in Definition 2.8, let $\mathfrak{a}=\left(a_{1}, a_{2}\right)$ be a multiweight for $X$. Suppose that $u \in \mathcal{A}^{\mathfrak{a}}(X)$ is conormal on $X$ with multiweight $\mathfrak{a}$. Then $u \in \mathcal{A}^{F_{1}, F_{2}}(X)$ if and only if for $(i, j)$ equal to $(1,2)$ or $(2,1)$, there exists a collar neighbourhood $[0,1)_{x_{i}} \times H_{i}$ of $H_{i}$ and functions $a_{z, p} \in \mathcal{A}_{\mathrm{phg}}^{F_{j}}\left(H_{i}\right),(z, p) \in F_{i}$, polyhomogeneous conormal with respect to $F_{j}$ at $\partial H_{i}=H_{i} \cap H_{j}$, such that for all $N \in \mathbb{N}$,

$$
u-\sum_{(z, p) \in F_{i}, \operatorname{Re}} a_{z \leq N}(y) x_{i}^{z}\left(\log x_{i}\right)^{p}=o\left(x_{i}^{N} x_{j}^{a_{j}}\right) .
$$

Proof. Apply the argument in the proof of Proposition 2.9 to the expansion at $H_{1}$ and at $\mathrm{H}_{2}$.

Definition 2.11 (Full $b$-calculus). The full b-calculus $\Psi_{b}^{m, \varepsilon}$ on $X$, where $m$ is a real number and $\mathcal{E}=\left(E_{\mathrm{lbz}}, E_{\mathrm{rbz}}\right)$ is an index family for $X^{2}$, is defined as follows. A half-density distribution $u(\kappa \otimes \kappa)$ on $X_{b}^{2}$ is in $\Psi_{b}^{m, \mathcal{E}}(X)$ if and only if $u=u_{1}+u_{2}+u_{3}$, where

(i) $u_{1}(\kappa \otimes \kappa)$ is in the small calculus $\Psi_{b}^{m}$;

(ii) $u_{2}$ is polyhomogeneous conormal with respect to the index family $\left(E_{\mathrm{lbz}}\right.$, $\left.C^{\infty}, E_{\mathrm{rbz}}\right)$, where $C^{\infty}:=\left\{(n, 0): n \in \mathbb{N}_{0}\right\}$ is the $C^{\infty}$ index set, and the index sets $E_{\mathrm{lbz}}, C^{\infty}$, and $E_{\mathrm{rbz}}$ are assigned to the three boundary hypersurfaces lbz, zf, and rbz respectively;

(iii) $u_{3}=\beta^{*} v$, where $\beta: X_{b}^{2} \rightarrow X^{2}$ is the blow-down map and $v$ is polyhomogeneous conormal with respect to the index family $\mathcal{E}$.

Proposition 2.12 (Proposition 5.46 in [37]). The full b-calculus on $X$ is a twosided module over the small b-calculus, i.e.,

$$
\Psi_{b}^{m, \varepsilon}(X) \circ \Psi_{b}^{m^{\prime}}(X) \subset \Psi_{b}^{m+m^{\prime}, \mathcal{\varepsilon}}(X),
$$

and

$$
\Psi_{b}^{m^{\prime}}(X) \circ \Psi_{b}^{m, \mathcal{E}}(X) \subset \Psi_{b}^{m+m^{\prime}, \mathcal{E}}(X),
$$

where $m, m^{\prime} \in \mathbb{R}$, and $\mathcal{E}$ is an index family.

The reason to introduce the full $b$-calculus is that, within it, we can construct parametrices of elliptic $b$-differential operators with compact error term. For the proof of the following proposition, see Proposition 5.59 in [37]. 
Proposition 2.13. Let $P$ be an elliptic b-differential operator of order $k$ whose indicial operator $I_{b}(P)$ is invertible on $L^{2}(\partial X \times \mathbb{R})$. Then there exists $G$ in the full b-calculus of order $-k$ such that the Schwartz kernels of the error terms $E=$ $\mathrm{Id}-P G$ and $E^{\prime}=\mathrm{Id}-G P$ are smooth across the diagonal, vanish at $\mathrm{zf}$, and are polyhomogeneous conormal at $\mathrm{lbz}$ and $\mathrm{rbz}$ with positive orders of vanishing there. This implies that $E$ and $E^{\prime}$ are compact on $L^{2}(X)$. Necessarily (in view of Proposition 2.5), we have

$$
I_{b}(G)=I_{b}(P)^{-1}
$$

\subsection{Scattering calculus}

Let $X$ be a manifold with boundary $\partial X$ and with local coordinates $x, y_{1}, \ldots, y_{d-1}$ near $\partial X$, where $x$ is a boundary defining function for $\partial X$. A smooth vector field $V$ on $X$ is a scattering vector field if it is $x$ multiplied by a $b$-vector field on $X$, i.e., it has the form

$$
V=a_{0} x^{2} \partial_{x}+a_{1} x \partial_{y_{1}}+\cdots+a_{d-1} x \partial_{y_{d-1}},
$$

where the coefficients $a_{0}, \ldots, a_{d-1}$ are smooth functions of $x$ and $y$. Written in terms of $r=x^{-1}$, these take the form

$$
V=-a_{0} \partial_{r}+\frac{a_{1}}{r} \partial_{y_{1}}+\cdots+\frac{a_{d-1}}{r} \partial_{y_{d-1}} .
$$

A scattering differential operator is one that is generated over $C^{\infty}(X)$ by scattering vector fields. A key example is when $X$ is the radial compactification of $\mathbb{R}^{d}$; then any constant coefficient vector field on $\mathbb{R}^{d}$ is a scattering vector field viewed on $X$, and therefore any constant coefficient differential operator on $\mathbb{R}^{d}$ is a scattering differential operator on $X$. The idea of the scattering calculus is to 'microlocalize' this set of differential operators.

To define it we first need to blow up the product $X^{2}$ to produce the scattering double space. This is done in two stages. The first is to create the $b$-double space $X_{b}^{2}=\left[X^{2} ;(\partial X)^{2}\right]$ as in the previous subsection. After this blowup, the diagonal lifts to be a product-type submanifold in $X_{b}^{2}$, i.e., can be expressed as the vanishing of $d$ coordinates in a coordinate system. The second step is to blow up the boundary of the lifted diagonal. The new boundary hypersurfaces so created are denoted bf and sf, respectively. Notice that in $X_{b}^{2}$, the lifted diagonal is naturally diffeomorphic to $X$, hence its boundary is naturally diffeomorphic to $\partial X$. So sf is a bundle over $\partial X$, with $n$-dimensional fibres. In fact, more is true.

Proposition 2.14. The interior of each fibre of the scattering face sf in the scattering double space $X_{\mathrm{sc}}^{2}$ has a natural vector space structure. Moreover, any scattering vector field lifts from either the left or the right factor to be tangent to sf, and to be a constant coefficient vector field on each fibre.

For the proof, see [34]. It is convenient to regard elements of the scattering calculus (defined in the next paragraph) as acting on scattering half-densities. We 
define a smooth scattering half-density on $X$ to be a smooth half-density in the interior of $X$ taking the form $f\left|r^{d-1} d r d y\right|^{1 / 2}, f \in C^{\infty}(X)$ at the boundary. We say that a smooth scattering half-density is nonvanishing if it is nonvanishing in the interior of $X$ and if $f>0$ everywhere. Let $\nu_{\mathrm{sc}}$ be a fixed nonvanishing smooth scattering half-density, and let $\nu_{\mathrm{sc}} \otimes \nu_{\mathrm{sc}}$ be the tensor product of $\nu_{\mathrm{sc}}$ in each of the left and right factors of $X$, lifted to $X_{\mathrm{sc}}^{2}$.

Definition 2.15 (Melrose, [38]). An element $A$ of the scattering calculus $\Psi_{\mathrm{sc}}^{m, l}(X)$ of order $(m, l)$ is a distribution $v$ on $X_{\mathrm{sc}}^{2}$ times $\nu_{\mathrm{sc}} \otimes \nu_{\mathrm{sc}}$, satisfying

(i) $x^{-l} v$ is conormal of order $m$, in the sense of Definition 18.2.6 and Theorem 18.2.8 in [32], with respect to the diagonal (more precisely the diagonal lifted to $X_{\mathrm{sc}}^{2}$ ) uniformly up to sf, where $x$ is a boundary defining function for sf;

(ii) $v$ vanishes to infinite order at the other boundary hypersurfaces.

The order $m$ is called the differential order of $v$, and $l$ the boundary order.

Remark 2.16. Using the Schwartz kernel theorem, elements of $\Psi_{\mathrm{sc}}^{m, l}(X)$ may be interpreted as operators on half-densities on $X$. A scattering differential operator of order $m$ acting on half-densities is in $\Psi_{\mathrm{sc}}^{m, 0}(X)$.

The scattering calculus is closed under composition.

Proposition 2.17 (Eqn. 6.12 in [39]). Let $X$ be a manifold with boundary, and $m, l, m^{\prime}, l^{\prime} \in \mathbb{R}$. Then

$$
\Psi_{\mathrm{sc}}^{m, l}(X) \circ \Psi_{\mathrm{sc}}^{m^{\prime}, l^{\prime}}(X) \subset \Psi_{\mathrm{sc}}^{m+m^{\prime}, l+l^{\prime}}(X) .
$$

Moreover, elements of $\Psi_{\mathrm{sc}}^{0,0}(X)$ act as bounded operators on $L^{2}$ half-densities.

Like Proposition 2.3 on the parametrix constructions under the small $b$-calculus, under the scattering calculus we also have a result analogous to Theorem 18.1.24 in $[32]$.

Proposition 2.18. Suppose that $P \in \Psi_{\mathrm{sc}}^{k, 0}(X)$ is elliptic. Then there exists $G \in$ $\Psi_{\mathrm{sc}}^{-k, 0}(X)$ such that

$$
P G-\mathrm{Id}, \quad G P-\mathrm{Id} \in \Psi_{\mathrm{sc}}^{-\infty, 0}(X) .
$$

Similarly to the case of the indicial operators in Section 2.1, the normal operator of $A \in \Psi^{m, 0}(X)$, denoted $N_{\mathrm{sc}}(A)$, is defined to be the restriction of the Schwartz kernel of $A$ to the scattering face sf. This restriction can be interpreted (in a canonical way) as a smooth function on sf valued in densities on each fibre. These densities can be interpreted as convolution operators on functions (or halfdensities) on each fibre (recall that, according to Proposition 2.14, each fibre is a vector space, so convolution is well defined). Under this interpretation, normal operators can be composed, and the action of taking normal operators is an algebra homomorphism:

Proposition 2.19 (Eqn. 5.14 in [38]). Let $A$ and $B$ be elements of $\Psi^{*, 0}(X)$. Then

$$
N_{\mathrm{sc}}(A B)=N_{\mathrm{sc}}(A) N_{\mathrm{sc}}(B) \text {. }
$$


As with the indicial operator, vanishing of the normal operator is related to compactness:

Proposition 2.20. Let $A \in \Psi^{m, 0}(X)$ with $m<0$. Then $A$ is compact if and only if $N_{\mathrm{sc}}(A)$ vanishes identically.

Remark 2.21. Alternatively, we may describe the boundary behaviour in the scattering calculus by taking the fibrewise Fourier transform of each convolution operator, obtaining a family of multipliers; this is known as the normal or boundary symbol. Composition in terms of the boundary symbol is given simply by the pointwise product.

Proposition 2.22. If $A \in \Psi^{m, 0}(X)$ is elliptic with invertible normal operator, then there exists $B \in \Psi^{-m, 0}(X)$ such that $E=A B-\operatorname{Id}$ is in $\Psi^{-\infty, \infty}(X)$, i.e., its Schwartz kernel is smooth across the diagonal and rapidly vanishing at the boundary of $X_{\mathrm{sc}}^{2}$. In particular, $E$ is compact and hence $A$ is Fredholm, with parametrix $B$. Necessarily, we have

$$
N_{\mathrm{sc}} B=\left(N_{\mathrm{sc}} A\right)^{-1} \text {. }
$$

Proof. See Section 6 of [38].

\section{The blown-up double space $M_{b, \mathrm{sc}}^{2}$}

As discussed in the introduction, we will construct the resolvent kernel $P^{-1}=$ $(H+1)^{-1}$ on a compactified and blown-up version of its natural domain $M^{2}$, using both $b$-blowups and scattering blowups. We start by compactifying $M^{2}$ in each factor separately, i.e., we pass to the compact space $[0, \infty]_{r} \times Y \times[0, \infty]_{r^{\prime}} \times Y$, where $[0, \infty]$ indicates the compactification of $[0, \infty)$ by a point at infinity, such that $1 / r$ is a boundary defining function at $r=\infty$. As noted in the introduction, $r P r$ is an elliptic $b$-differential operator near $r=0$, while $P$ itself is an elliptic scattering differential operator as $r \rightarrow \infty$. Therefore we perform the $b$-blowup at $r=r^{\prime}=0$ and the scattering blowup at $r=r^{\prime}=\infty$. This means that we blow up the corner $r=r^{\prime}=0$, the corner $r=r^{\prime}=\infty$, and finally the boundary of the lifted diagonal $\left\{r=r^{\prime}, y=y^{\prime}\right\}$ at $r=r^{\prime}=\infty$.

We label the boundary hypersurfaces of $[0, \infty]_{r} \times Y \times[0, \infty]_{r^{\prime}} \times Y$ by lbz, lbi, rbz, and rbi according to whether they arise from $\{r=0\},\{r=\infty\},\left\{r^{\prime}=0\right\}$, or $\left\{r^{\prime}=\infty\right\}$, respectively. The new boundary hypersurfaces created by blowup are labelled $\mathrm{zf}$, bf, and sf, according to whether they arise from the blowup of $r=r^{\prime}=0, r=r^{\prime}=\infty$, or the boundary of the lifted diagonal at $r=r^{\prime}=\infty$, respectively. The resulting space after the blowups at $r=r^{\prime}=0$, and $r=r^{\prime}=\infty$ is denoted by $M_{b, \mathrm{sc}}^{2}$. See Figure 1 .

We next discuss local coordinates near the various blown-up faces. Near zf, local coordinates are $\left(r / r^{\prime}, r^{\prime}, y, y^{\prime}\right)$ when $r / r^{\prime} \leq C$ (that is, away from rbz) and $\left(r, r^{\prime} / r, y, y^{\prime}\right)$ when $r^{\prime} / r \leq C$ (that is, away from lbz). Near bf and away from sf the situation is similar; coordinates are $\left(r^{\prime} / r, r^{\prime-1}, y, y^{\prime}\right)$ for $r^{\prime} / r \leq C$ and $\left(r / r^{\prime}, r^{-1}, y, y^{\prime}\right)$ for $r / r^{\prime} \leq C$. Near the interior of sf, coordinates are $\left(r-r^{\prime}, r\left(y-y^{\prime}\right), y, r^{-1}\right)$. 
In the case that $M$ is Euclidean space $\mathbb{R}^{d}$, with Euclidean coordinate $z$, then $z-z^{\prime}$ is a linear coordinate on each fibre of sf (recall by Proposition 2.14, the fibres of sf have a linear structure). In particular, the diagonal is defined by $r / r^{\prime}=1, y=y^{\prime}$ for small $r$ (that is, away from sf ) and $r-r^{\prime}=0, r\left(y-y^{\prime}\right)=0$ or $r-r^{\prime}=0, r^{\prime}\left(y-y^{\prime}\right)=0$ for large $r$ (that is, away from zf). The following result about the diagonal will be useful later.

Proposition 3.1. Let $\varphi:[0, \infty) \rightarrow[0,1]$ be an increasing smooth function such that $\varphi(x)=x$ for $x \in[0,1 / 2]$ and $\varphi(x)=1$ for $x \in[1, \infty)$. Then the function

$$
a_{\operatorname{diag}}\left(z, z^{\prime}\right)=\frac{d\left(z, z^{\prime}\right)^{2}}{\varphi^{2}\left(r^{\prime}\right)}
$$

where $z=(r, y)$ and $z^{\prime}=\left(r^{\prime}, y^{\prime}\right)$, is a quadratic defining function for the diagonal in $M_{b, \mathrm{sc}}^{2}$; that is, $a_{\mathrm{diag}} \geq 0$, the diagonal lifted to the blown up space is given by $\left\{a_{\mathrm{diag}}=0\right\}$, and the Hessian of $a_{\mathrm{diag}}$ in directions normal to the lifted diagonal is positive definite.

Proof. The formula for the distance on a metric cone is given by

$$
d\left(z, z^{\prime}\right)^{2}= \begin{cases}r^{2}+r^{\prime 2}-2 r r^{\prime} \cos \left(d_{Y}\left(y, y^{\prime}\right)\right), & d_{Y}\left(y, y^{\prime}\right) \leq \pi \\ \left(r+r^{\prime}\right)^{2}, & d_{Y}\left(y, y^{\prime}\right) \geq \pi\end{cases}
$$

(The second line is because when $d_{Y}\left(y, y^{\prime}\right) \geq \pi$ the fastest way to get from $(r, y)$ to $\left(r^{\prime}, y^{\prime}\right)$ is to go straight to the cone point and back out again.) So near the diagonal we have

$$
\begin{aligned}
d\left(z, z^{\prime}\right)^{2} & =\left(r-r^{\prime}\right)^{2}+2 r r^{\prime}\left(1-\cos \left(d_{Y}\left(y, y^{\prime}\right)\right)\right) \\
& =\left(r-r^{\prime}\right)^{2}+r r^{\prime}\left(d_{Y}\left(y, y^{\prime}\right)^{2}+O\left(d_{Y}\left(y, y^{\prime}\right)^{4}\right)\right) .
\end{aligned}
$$

Near the sf-face, we have

$$
a_{\mathrm{diag}}\left(z, z^{\prime}\right)=d\left(z, z^{\prime}\right)^{2}=\left(r-r^{\prime}\right)^{2}+r r^{\prime}\left(d_{Y}\left(y, y^{\prime}\right)^{2}+O\left(d_{Y}\left(y, y^{\prime}\right)^{4}\right)\right),
$$

which is a quadratic defining function for the diagonal. To see that we recall from the discussion before this proposition that near sf the diagonal is defined by $r^{\prime}-r=0$, and $r\left(y-y^{\prime}\right)=0$ or $r^{\prime}\left(y-y^{\prime}\right)=0$, and we also recall the standard fact that $d_{Y}\left(y, y^{\prime}\right)^{2}$ is a quadratic defining function for the diagonal of $Y^{2}$ for any closed Riemannian manifold $Y$.

Near the zf-face, we have

$$
a_{\mathrm{diag}}\left(z, z^{\prime}\right)=\frac{d\left(z, z^{\prime}\right)^{2}}{r^{\prime 2}}=\left(\frac{r}{r^{\prime}}-1\right)^{2}+\frac{r}{r^{\prime}}\left(d_{Y}\left(y, y^{\prime}\right)^{2}+O\left(d_{Y}\left(y, y^{\prime}\right)^{4}\right)\right),
$$

which is again a quadratic defining function for the diagonal, as near zf the diagonal is defined by $r / r^{\prime}=1$ and $y=y^{\prime}$.

Proposition 1 from Section 4, Chapter VI of [43] immediately implies the following. 
Lemma 3.2. Let $K\left(z, z^{\prime}\right)$ be a Schwartz kernel on $M_{b, \mathrm{sc}}^{2}$, conormal (in the sense of Definitions 2.1 and 2.15) of order $m$ at the lifted diagonal, with $m \geq-d$. Then $K$ satisfies

$$
\left|K\left(z, z^{\prime}\right)\right| \leq C a_{\operatorname{diag}}^{-(d+m) / 2}, \quad z \neq z^{\prime}
$$

for some constant $C$.

\subsection{Densities on $M_{b, \mathrm{sc}}^{2}$}

By a smooth $b$-half-density on $M_{b, \mathrm{sc}}^{2}$ we mean a half-density of the form

$$
u\left(r, r^{\prime}, y, y^{\prime}\right)\left|\frac{d r}{r} \frac{d r^{\prime}}{r^{\prime}} d y d y^{\prime}\right|^{1 / 2},
$$

where $u$ is smooth. (This is perhaps misleading since it is only a $b$-half density in the usual sense away from sf. However, we shall only use this when either $r$ or $r^{\prime}$ is small, in which case it certainly is a $b$-half density.) Let $x=1 / r$ and $x^{\prime}=1 / r^{\prime}$. Then by a smooth scattering half-density we mean a density of the form,

$$
v\left(x, x^{\prime}, y, y^{\prime}\right)\left|\frac{d x d x^{\prime} d y d y^{\prime}}{x^{d+1} x^{\prime d+1}}\right|^{1 / 2},
$$

where $v$ is smooth. In terms of $r$ and $r^{\prime}$ it becomes,

$$
v\left(r, r^{\prime}, y, y^{\prime}\right)\left|r^{d+1} r^{\prime d+1} d\left(\frac{1}{r}\right) d\left(\frac{1}{r^{\prime}}\right) d y d y^{\prime}\right|^{1 / 2}=v\left(r, r^{\prime}, y, y^{\prime}\right)\left|r^{d-1} r^{\prime d-1} d r d r^{\prime} d y d y^{\prime}\right|^{1 / 2} .
$$

The scattering half-density $\left|r^{d-1} r^{\prime d-1} d r d r^{\prime} d y d y^{\prime}\right|^{1 / 2}$ is a bounded nonzero multiple of the Riemannian half-density. We will usually consider the resolvent $P^{-1}$ as acting on Riemannian half-densities, in which case the kernel of $P^{-1}$ itself is a Riemannian (distributional) half-density on $M_{b, \mathrm{sc}}^{2}$. However, when we study the properties of a kernel near the zf-face, we write it as a $b$-half-density; this is more natural in view of the fact that we use the $b$-calculus near zf.

\section{Resolvent construction}

Let $Y, M, \Delta, V_{0}, H=H_{V_{0}}$, and $T=T_{V_{0}}$ be as in the introduction. We assume throughout that $V_{0}$ satisfies condition (1.3). In this section we construct the inverse of the operator $H+1$ and investigate properties of its Schwartz kernel.

\subsection{Positivity of $H$}

Proposition 4.1. Suppose that $V_{0}$ satisfies (1.3). Then the operator $H$ is also positive.

Proof. We work in polar coordinates. Consider the isometry $U$ mapping from $L^{2}\left(M ; r^{d-1} d r d y\right)$ to $L^{2}\left(M ; r^{-1} d r d y\right)$ defined by

$$
U f=r^{d / 2} f \text {. }
$$


Now for $f \in L^{2}\left(M ; r^{-1} d r d y\right)$, we compute

$$
\begin{aligned}
U H U^{-1} f & =r^{d / 2}\left(-\partial_{r}^{2}-\frac{d-1}{r} \partial_{r}+\frac{1}{r^{2}} \Delta_{Y}+\frac{V_{0}(y)}{r^{2}}\right) r^{-d / 2} f \\
& =\left(\frac{d(d-4)}{4}+V_{0}(y)\right) \frac{1}{r^{2}} f+\frac{1}{r} \partial_{r} f-\partial_{r}^{2} f+\frac{1}{r^{2}} \Delta_{Y} f .
\end{aligned}
$$

A short computation shows that

$$
\begin{aligned}
\frac{1}{r}\left(-\left(r \partial_{r}\right)^{2}\right. & \left.+\Delta_{Y}+\left(\frac{d-2}{2}\right)^{2}+V_{0}(y)\right) \frac{f}{r} \\
& =-\frac{1}{r^{2}} f+\frac{1}{r} \partial_{r} f-\partial_{r}^{2} f+\frac{1}{r^{2}} \Delta_{Y} f+\left(\left(\frac{d-2}{2}\right)^{2}+V_{0}(y)\right) \frac{1}{r^{2}} f \\
& =\left(\frac{d(d-4)}{4}+V_{0}(y)\right) \frac{1}{r^{2}} f+\frac{1}{r} \partial_{r} f-\partial_{r}^{2} f+\frac{1}{r^{2}} \Delta_{Y} f=U H U^{-1} f .
\end{aligned}
$$

We have thus established

$$
U H U^{-1}=\frac{1}{r}\left(-\left(r \partial_{r}\right)^{2}+\Delta_{Y}+V_{0}(y)+\left(\frac{d-2}{2}\right)^{2}\right) \frac{1}{r} .
$$

Make the substitution $s=\ln r$. Then the space $L^{2}\left(M ; r^{-1} d r d y\right)$ becomes $L^{2}(M$; $d s d y$ ), and we have

$$
U H U^{-1}=e^{-s}\left(-\partial_{s}^{2}+\Delta_{Y}+V_{0}(y)+\left(\frac{d-2}{2}\right)^{2}\right) e^{-s} .
$$

From here we can clearly see that the operator $H$ is positive if $\Delta_{Y}+V_{0}(y)+$ $((d-2) / 2)^{2}>0$. This completes the proof.

\subsection{The Riesz transform $T$}

Our aim is to find the precise range of $p$ for which the Riesz transform $T=$ $\nabla H^{-1 / 2}$ is bounded on $L^{p}(M)$. Following [14] and [27], we do this using a 'resolvent approach' as opposed to the more common 'heat kernel approach'. Using functional calculus, we have the following expression,

$$
T=\frac{2}{\pi} \int_{0}^{\infty} \nabla\left(H+\lambda^{2}\right)^{-1} d \lambda .
$$

We see from this equation that in order to understand $T$, we need to know the properties of $\left(H+\lambda^{2}\right)^{-1}$. Because $H$ is homogeneous of degree -2 , we only need to compute $(H+1)^{-1}$, then use scaling. Let $P=H+1$; we proceed to study $P^{-1}$.

\subsection{A formula for the resolvent}

We now proceed to find an explicit formula for $P^{-1}$. However as we will discuss later, the formula has good convergence properties in only certain regions of $M_{b, \mathrm{sc}}^{2}$. From equation (4.2) we have

$$
P=H+1=r^{-d / 2-1}\left(-\left(r \partial_{r}\right)^{2}+\Delta_{Y}+V_{0}(y)+r^{2}+\left(\frac{d-2}{2}\right)^{2}\right) r^{d / 2-1} .
$$


Let $P^{\prime}$ denote the differential operator consisting of the terms in the middle. That is,

$$
P^{\prime}=-\left(r \partial_{r}\right)^{2}+\Delta_{Y}+V_{0}(y)+r^{2}+\left(\frac{d-2}{2}\right)^{2}
$$

We take $P^{\prime}$ to act on half-densities, using the flat connection that annihilates the Riemannian half-density $\left|r^{d-1} d r d h\right|^{1 / 2}$ on $M$. Now let $\tilde{P}$ be the differential operator given by the same expression (4.3), but endowed with the flat connection on half-densities annihilating the $b$-half density $|d r / r d h|^{1 / 2}$. Since $U$ maps this $b$-half density to the Riemannian half-density, these two differential operators are related by

$$
\tilde{P}=U^{-1} P^{\prime} U
$$

Therefore,

$$
P=r^{-1} \tilde{P} r^{-1}
$$

Since $P$ is self-adjoint, equation (4.5) shows that $\tilde{P}$ is also self-adjoint. (Note that for operators on half-densities there is an invariant notion of self-adjointness, since the inner product on half-densities is invariantly defined.) Write $G=P^{-1}$ and $\tilde{G}=\tilde{P}^{-1} ;$ the Schwartz kernels of $G$ and $\tilde{G}$ are related by

$$
G=r r^{\prime} \tilde{G}
$$

Again, we emphasize that this is an identity involving half-densities; if we write the half-densities $G$ and $\tilde{G}$ as $K\left|\left(r r^{\prime}\right)^{d-1} d r d r^{\prime} d h d h^{\prime}\right|^{1 / 2}$ and $\tilde{K}\left|\left(r r^{\prime}\right)^{-1} d r d r^{\prime} d h d h^{\prime}\right|^{1 / 2}$ then we have

$$
K=\left(r r^{\prime}\right)^{1-d / 2} \tilde{K}
$$

So we just need to determine $\tilde{G}$, then equation (4.6) gives us $G$.

We now proceed to work out an expression for $\tilde{G}$. Let $\left(\mu_{j}^{2}, u_{j}\right)$ be the eigenvalues and corresponding $L^{2}$-normalized eigenfunctions of the positive operator $\Delta_{Y}+$ $V_{0}(y)+((d-2) / 2)^{2}$. We also let $\Pi_{j}$ denote the projection onto the $u_{j}$-eigenspace. Then we have

$$
\tilde{P}=\sum_{j} \Pi_{j} \tilde{T}_{j},
$$

and

$$
\mathrm{Id}=\sum_{j} \delta\left(\frac{r}{r^{\prime}}-1\right) \Pi_{j},
$$

where

$$
\tilde{T}_{j}=-\left(r \partial_{r}\right)^{2}+r^{2}+\mu_{j}^{2}=-r^{2} \partial_{r}^{2}-r \partial_{r}+\mu_{j}^{2}
$$


As in [27], the kernel of the inverse of $\tilde{T}_{j}$ is written in terms of modified Bessel functions $I_{\mu_{j}}(r)$ and $K_{\mu_{j}}(r)$ (see Section 9.6 of [1]) in the form

$$
\tilde{T}_{j}^{-1}\left(r, r^{\prime}\right)= \begin{cases}I_{\mu_{j}}(r) K_{\mu_{j}}\left(r^{\prime}\right)\left|\frac{d r}{r} \frac{d r^{\prime}}{r^{\prime}}\right|^{1 / 2}, & r<r^{\prime}, \\ K_{\mu_{j}}(r) I_{\mu_{j}}\left(r^{\prime}\right)\left|\frac{d r}{r} \frac{d r^{\prime}}{r^{\prime}}\right|^{1 / 2}, & r>r^{\prime} .\end{cases}
$$

We know that

$$
\tilde{G}=\sum_{j} \Pi_{j} \tilde{T}_{j}^{-1} .
$$

Hence, in terms of the kernels, we have

$$
\tilde{G}\left(r, r^{\prime}, y, y^{\prime}\right)= \begin{cases}\sum_{j} u_{j}(y) \overline{u_{j}\left(y^{\prime}\right)} I_{\mu_{j}}(r) K_{\mu_{j}}\left(r^{\prime}\right)\left|\frac{d r}{r} \frac{d r^{\prime}}{r^{\prime}} d h d h^{\prime}\right|^{1 / 2}, \quad r<r^{\prime}, \\ \sum_{j} u_{j}(y) \overline{u_{j}\left(y^{\prime}\right)} K_{\mu_{j}}(r) I_{\mu_{j}}\left(r^{\prime}\right)\left|\frac{d r}{r} \frac{d r^{\prime}}{r^{\prime}} d h d h^{\prime}\right|^{1 / 2}, \quad r>r^{\prime},\end{cases}
$$

where $d h$ denotes the Riemannian density with respect to the metric on $Y$. While this is an exact expression for $\tilde{G}$, it is not a very useful expression near the diagonal, as it has poor convergence properties. Therefore we shall glue it together with a pseudodifferential-type parametrix in order to determine its properties close to the diagonal. However, sufficiently far from the diagonal, the series has very good convergence. We proceed to show this.

Remark 4.2. We could consider self-adjoint extensions other than the Friedrichs extension for the Laplacian acting on $C_{c}^{\infty}(Y \times(0, \infty))$. If we do, then the form of the inverse in (4.10) changes; in particular, the Bessel function $I_{\mu_{j}}$ would be replaced by a certain linear combination of $I_{\mu_{j}}$ and $K_{\mu_{j}}$ in formula (4.10), depending on the extension. (Indeed, the Friedrichs extension is the only one for which the domain of the extension is contained in the form domain, requiring the derivatives of $\tilde{T}_{j}^{-1}\left(r, r^{\prime}\right)$ to be in $L^{2}(d r / r)$ as $r \rightarrow 0$ for fixed $r^{\prime}$ and vice versa.) This would have the effect of changing the order of vanishing of the Riesz transform at the lbz and rbz boundary hypersurfaces, and thus the range of $p$ for which one has boundedness on $L^{p}$ (see the proofs of Propositions 5.10 and 5.12). Thus our results only hold for the Friedrichs extension.

\subsection{Convergence of the formula}

By the symmetry of (4.11), it suffices to consider the region $\left\{r<r^{\prime}\right\}$; here we work with the sum

$$
\sum_{j} u_{j}(y) \overline{u_{j}\left(y^{\prime}\right)} I_{\mu_{j}}(r) K_{\mu_{j}}\left(r^{\prime}\right) .
$$

In the appendix we prove the following estimates on the functions $I_{\mu}$ and $K_{\mu}$. 
Proposition 4.3. 1) The functions $I_{\mu}$ and $K_{\mu}$ satisfy the following estimates. First, the derivatives of order $l, 0 \leq l \leq \mu$, satisfy

$$
\begin{aligned}
\left|\left(\frac{d}{d r}\right)^{l} I_{\mu}(r)\right| & \leq C_{l} \mu^{l} \frac{2^{-\mu} r^{\mu-l} e^{r}}{\Gamma(\mu+1 / 2)} \\
\left|\left(\frac{d}{d r}\right)^{l} K_{\mu}(r)\right| & \leq C_{l} \mu^{l} 2^{3 \mu} \Gamma(\mu) r^{-\mu-l} e^{-r / 2}
\end{aligned}
$$

for some constant $C_{l}$ depending only on $l$.

2) The functions $I_{\mu}$ and $K_{\mu}$ have expansions at $r=0$ of the form

$$
I_{\mu}(r)=\frac{r^{\mu} 2^{-\mu}}{\Gamma(\mu+1 / 2)}\left(\sum_{n=0}^{N-1} a_{n, \mu} r^{2 j}+O\left(r^{2 N}\right)\right), \quad r \leq 1
$$

where the $a_{n, \mu}$ are uniformly bounded in both $n$ and $\mu$. Similarly, for $N<\mu$ the function $K_{\mu}$ has an expansion

$$
K_{\mu}(r)=r^{-\mu} 2^{3 \mu} \Gamma(\mu)\left(\sum_{n=0}^{N-1} b_{n, \mu} r^{2 j}+O\left(r^{2 N}\right)\right), \quad r \leq 1,
$$

where the $b_{n, \mu}$ are uniformly bounded in both $n$ and $\mu$ for $n<\mu-1$. Moreover, the implied constants $C_{N, \mu}$ in the error terms $O\left(r^{2 N}\right)$ in expansions (4.14) and (4.15) can be taken to be independent of $\mu$, provided $N<\mu$.

We will also use the following lemma proved in the appendix:

Lemma 4.4. Suppose that $\mu_{j}^{2}$ are the eigenvalues of $\Delta_{Y}+V_{0}(y)+((d-2) / 2)^{2}$, with $\mu_{j}>0$. Then for any $0<\beta<1$, and any $M, N \geq 0$, the sum

$$
\sum_{\mu_{j} \geq M} \mu_{j}^{N} \alpha^{\mu_{j}-M}
$$

converges for all $0<\alpha \leq \beta$, and it is bounded uniformly in $\alpha$.

Using these results we show:

Proposition 4.5. For $r^{\prime}>4 r$ or $r>4 r^{\prime}$ the series (4.12) converges, and the sum is polyhomogeneous conormal on $M_{b, \mathrm{sc}}^{2}$ with index sets at $\mathrm{zf}, \mathrm{lbz}$, and $\mathrm{rbz}$ given by

$$
\begin{aligned}
& F_{\mathrm{zf}}=\mathbb{N}_{0} \times\{0\} \cup\left\{(z, 0): z=2 \mu_{j}+k, k \in \mathbb{N}_{0}\right\} \cup\left\{\left(2 \mu_{j}+k, 1\right): k \in \mathbb{N}_{0}, \mu_{j} \in \mathbb{N}_{0}\right\} \\
& F_{\mathrm{lbz}}=F_{\mathrm{rbz}}=\left\{(z, 0): z=\mu_{j}+k, k \in \mathbb{N}_{0}\right\}
\end{aligned}
$$

and all other index sets empty (meaning that the series vanishes rapidly together with all derivatives at the other boundary hypersurfaces of $M_{b, \mathrm{sc}}^{2}$ ). 
Proof. We consider only the case $r^{\prime}>4 r$; the other follows by symmetry.

It is well known that the functions $I_{\mu}$ and $K_{\mu}$ are polyhomogeneous conormal, with $I_{\mu}$ having index set $\left\{(\mu+k, 0): k \in \mathbb{N}_{0}\right\}$ and $K_{\mu}$ having index set

$$
\left\{(-\mu+k, 0): k \in \mathbb{N}_{0}\right\} \cup\left\{(\mu+k, 0): k \in \mathbb{N}_{0}\right\}
$$

if $\mu$ is not an integer, or

$$
\left\{(-\mu+k, 0): k \in \mathbb{N}_{0}\right\} \cup\left\{(\mu+k, 1): k \in \mathbb{N}_{0}\right\}
$$

if $\mu$ is an integer (see Equations 9.6.10, 9.6.2, and 9.6.11 in [1]). Moreover, $K_{\mu}(r)$ is exponentially decreasing as $r \rightarrow \infty$. This implies that $I_{\mu}(r) K_{\mu}\left(r^{\prime}\right)$ is, for $4 r \leq r^{\prime}$, polyhomogeneous on $M_{b, \mathrm{sc}}^{2}$ with index set $\left\{(\mu+k, 0) \mid k \in \mathbb{N}_{0}\right\}$ at lbz, the empty set (corresponding to rapid decrease) at rbi, and index set

$$
\left\{(k, 0): k \in \mathbb{N}_{0}\right\} \cup\left\{(2 \mu+k, 0): k \in \mathbb{N}_{0}\right\}
$$

if $\mu$ is not an integer, or

$$
\left\{(k, 0): k \in \mathbb{N}_{0}\right\} \cup\left\{(2 \mu+k, 1): k \in \mathbb{N}_{0}\right\}
$$

if $\mu$ is an integer, at zf. This implies that the $j$ th term in (4.12) is polyhomogeneous conormal with respect to the given index family (4.16); the issue is to show the same property for the sum of the series.

Applying (4.13) for $l=0$, we see that when $r^{\prime} \geq 4 r, I_{\mu}(r) K_{\mu}\left(r^{\prime}\right)$ is bounded above by

$$
C\left(\frac{4 r}{r^{\prime}}\right)^{\mu} e^{-r^{\prime} / 4}
$$

where $C$ is independent of $\mu$. By Hörmander's $L^{\infty}$-estimate, see [31], we know that $\left\|u_{j}\right\|_{\infty} \leq C \mu_{j}^{(d-1) / 2}$. Therefore each term in the series is bounded above by $C \mu_{j}^{d-1}\left(2 r / r^{\prime}\right)^{\mu_{j}} e^{-r^{\prime} / 4}$. Lemma 4.4, with $\alpha=r / r^{\prime}$, then shows that the series converges pointwise, and uniformly for $4 r / r^{\prime} \leq \beta<1$. More generally, applying b-derivatives (see Definition 2.6) to the series (4.12) and using the Hörmander estimate $\left\|\nabla^{(k)} u_{j}\right\|_{\infty} \leq C_{k} \mu_{j}^{(d-1) / 2+k}$ for derivatives of $u_{k}$, we see that the $l$ th bderivatives of the terms of the series (4.12) satisfy the same estimate except for an additional factor of $\mu^{l}$, which is harmless as Lemma 4.4 applies with arbitrary powers of $\mu$. Thus the series is (for $4 r \leq r^{\prime}$ ) conormal with weight 0 at zf, weight $\mu_{0}$ at $\mathrm{lbz}$, and vanishes rapidly at all other boundary hypersurfaces.

To prove polyhomogeneous conormality at lbz we use Proposition 2.10 showing that we only need to show that there is an expansion at lbz together with estimates on the remainder (with no need for derivative estimates). Observe that a boundary defining function $\rho_{\mathrm{lbz}}$ for $\mathrm{lbz}$ is comparable to $r / r^{\prime}$ for $r \leq 1$ and to $r$ for $r \geq 1$. Given $N$ we separate the series (4.12) into the terms with $\mu_{j} \leq N$ and the remainder. The first part is manifestly polyhomogeneous at lbz. On the other hand, we can pull out a factor of $\left(r / r^{\prime}\right)^{N}$ and apply Lemma 4.4 with $\alpha=r / r^{\prime}$ and $M=N$, showing that the sum of the remainder is $O\left(\left(r / r^{\prime}\right)^{N} e^{-r^{\prime} / 4}\right)$ which is $O\left(\rho_{\mathrm{lbz}}^{N}\right)$. This proves polyhomogeneous conormality at lbz, uniformly to the corner with zf. 
To prove polyhomogeneous conormality at $\mathrm{zf}$ is a bit more delicate as each term in (4.12) contributes to the leading term (and other terms) of the expansion of (4.12) at zf. We can take $r^{\prime}$ to be a boundary defining function for zf in the region $4 r \leq r^{\prime}$. Due to Proposition 2.10, it suffices to show that for each $N \in \mathbb{R}$, there is an expansion at zf as in (2.4) (taking $H_{i}=\mathrm{zf}, x_{i}=r^{\prime}, x_{j}=r / r^{\prime}$, and $\left.a_{j}=\mu_{0}\right)$ together with an $O\left(\left(r^{\prime}\right)^{N}\left(r / r^{\prime}\right)^{\mu_{0}}\right)$ estimate on the remainder.

Since we have already observed that each individual term in the sum (4.12) is polyhomogeneous with the required index family, given $N \in \mathbb{R}$, it suffices to consider the sum (4.12) for $\mu_{j}>N$. Using (4.14) and (4.15), the $j$ th term in this sum can be expanded as

$$
u_{j}(y) \overline{u_{j}\left(y^{\prime}\right)}\left(\frac{4 r}{r^{\prime}}\right)^{\mu_{j}} \frac{\Gamma\left(\mu_{j}\right)}{\Gamma\left(\mu_{j}+1 / 2\right)}\left(\sum_{m=0}^{N-1} \sum_{n=0}^{N-1} a_{m, \mu_{j}} b_{n, \mu_{j}}\left(\frac{r}{r^{\prime}}\right)^{2 m} r^{2 m+2 n}+O\left(r^{\prime 2 N}\right)\right)
$$

We are interested in summing this expression over all $\mu_{j}>N$ in the region $r / r^{\prime} \leq$ $1 / 4, r^{\prime} \leq 1$. Proposition 4.3 tells us that $a_{n, \mu}$ and $b_{n, \mu}$ are uniform for $n \leq N$. Using Lemma 4.4 we see that the sum over $\mu_{j} \geq N$ over the sum over $m$ and $n$ in (4.17) gives an expansion at zf involving even powers of $r^{\prime}$, up to $2 N$, with coefficients that are bounded at lbz. Next, we check that each fixed coefficient of this expansion at zf is polyhomogeneous conormal at lbz, using exactly the same argument as used above to show polyhomogeneous conormality globally at lbz. Finally, using Lemma 4.4 once more, and the last statement in Proposition 4.3, we check that the sum in $\mu_{j} \geq N$ over the error terms $O\left(r^{\prime 2 N}\right)$ in (4.17) is a term bounded by $C_{N}{r^{\prime}}^{2 N}\left(r / r^{\prime}\right)^{N}$. This completes the proof of polyhomogeneous conormality at zf.

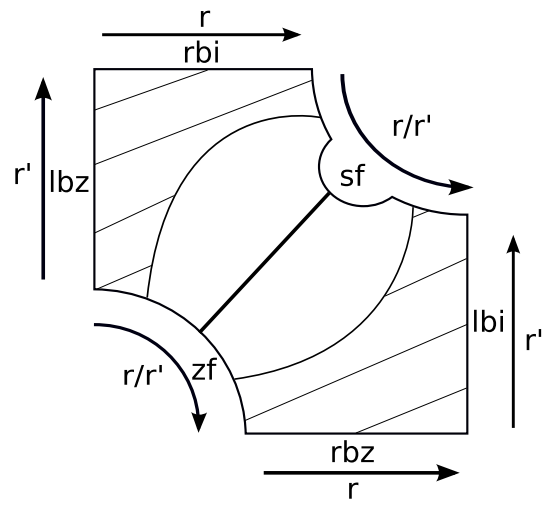

Figure 2. Support of $\tilde{G}_{f}$.

Proposition 4.5 implies, in particular, that $\tilde{G}$ decays exponentially, with all its derivatives, as $r^{\prime} \rightarrow \infty$, i.e., when approaching the boundary rbi. Similarly in the region $r / r^{\prime} \geq 4$, as $r \rightarrow \infty$, i.e., when approaching lbi, the kernel is also 
exponentially decreasing. Therefore we cut off $\tilde{G}$ to restrict it away from the $r=r^{\prime}$ to obtain a well defined operator $\tilde{G}_{f}$ with the kernel

$$
\tilde{G}_{f}\left(r, r^{\prime}, y, y^{\prime}\right)=\tilde{G}\left(r, r^{\prime}, y, y^{\prime}\right)\left(\chi\left(\frac{4 r}{r^{\prime}}\right)+\chi\left(\frac{4 r^{\prime}}{r}\right)\right) .
$$

Here $\chi$ is a smooth cutoff function $\chi:[0, \infty) \rightarrow[0,1]$ such that $\chi([0,1 / 2])=1$ and $\chi([1, \infty))=0$. Thus the support of $\tilde{G}_{f}$ is contained in $\left\{r / r^{\prime} \leq 1 / 4\right\} \cup\left\{r / r^{\prime} \geq 4\right\}$, as illustrated in Figure 2. (The subscript ' $f$ ' stands for 'far from the diagonal'.)

At last, similar to (4.6), we define

$$
G_{f}=r r^{\prime} \tilde{G}_{f}
$$

\subsection{Near diagonal}

The formula obtained in the previous section does not have good convergence properties near the diagonal, so in this section we construct an operator $G_{n d}$ which is a parametrix for $P$ near the diagonal. The subscript $n d$ means "near diagonal".

Near the zf-face we consider the $b$-elliptic operator $\tilde{P}$. In order to keep it away from the sf-face, we multiply it with a cutoff function, so we consider $\tilde{P} \chi(r)$, where $\chi:[0, \infty) \rightarrow[0,1]$ is a smooth cutoff function as above. By the ellipticity of $\tilde{P}$ near the zf-face, and by Proposition 2.13, there is $\tilde{G}_{n d}^{z f}$ in the full $b$-calculus such that

$$
\tilde{P} \tilde{G}_{n d}^{z f} \chi(r)=\chi(r)+\tilde{E}_{z f}
$$

where $\tilde{E}_{z f}$ is smooth across the diagonal and vanishes to first order at zf (as a $b$-half density). Let

$$
G_{n d}^{z f}=r r^{\prime} \tilde{G}_{n d}^{z f}
$$

Then we have

$$
P G_{n d}^{z f} \chi(r)=\chi(r)+E_{z f}
$$

where $E_{z f}=\left(r^{\prime} / r\right) \tilde{E}_{z f}$ is smooth across the diagonal and vanishes to first order at zf as a $b$-half density.

Near the sf-face the operator $P$ is elliptic in the scattering calculus. We multiply it with $1-\chi(r)$ to keep it away from the $z f$-face, i.e., we consider the operator $P(1-\chi(r))$. Since $P(1-\chi(r))$ is elliptic near the sf-face, and its normal operator $\Delta_{\mathbb{R}^{n}}+1$ is invertible, by Proposition 2.22 , there is $G_{n d}^{s f}$ in the scattering calculus such that

$$
P G_{n d}^{s f}(1-\chi(r))=1-\chi(r)+E_{s f},
$$

where the error term $E_{s f}$ is smooth across the diagonal and vanishes to infinite order at bf and sf.

Now we define $G_{n d}$ by

$$
G_{n d}=\left(G_{n d}^{z f} \chi(r)+G_{n d}^{s f}(1-\chi(r))\right)\left(1-\chi\left(\frac{4 r}{r^{\prime}}\right)-\chi\left(\frac{4 r^{\prime}}{r}\right)\right) .
$$


Then we have

$$
P G_{n d}=\mathrm{Id}+E_{n d}
$$

where the error term $E_{n d}$ is smooth across the diagonal, and vanishes to first order at zf (as a $b$-half density) and to infinite order at all other boundary hypersurfaces. We may assume that $G_{n d}$ is supported close to the union of the diagonal, zf, and sf.

We now define our global parametrix to be

$$
G_{a}=G_{f}+G_{n d}
$$

\subsection{The indicial operator at $\mathrm{zf}$}

In this subsection we show that the leading behaviour of $G_{a}$ at zf agrees with that of $G_{n d}^{z f}$. To do this, it suffices to show that the indicial operator of $\tilde{G}$ agrees (at least for $r / r^{\prime}<1 / 4$ and $r / r^{\prime}>4$, where we have shown convergence of the series) with that of $\tilde{G}_{n d}^{z f}$. By Proposition 2.13, the indicial operator of $\tilde{G}_{n d}^{z f}$ is equal to $I_{b}(\tilde{P})^{-1}$. Let us now determine this indicial operator.

The indicial operator of $\tilde{P}$ is

$$
I_{b}(\tilde{P} \chi(r))=-\left(r \partial_{r}\right)^{2}+\Delta_{Y}+V_{0}(y)+\left(\frac{d-2}{2}\right)^{2} .
$$

Let $\mu_{j}^{2}, u_{j}$, and $\Pi_{j}$ be defined as in Section 4.3. Here, instead of (4.8) and (4.9) we have

$$
I_{b}(\tilde{P} \chi(r))=\sum_{j} \Pi_{j} S_{j}
$$

where

$$
S_{j}=-\left(r \partial_{r}\right)^{2}+\mu_{j}^{2}
$$

As in Section 4.3, the kernel $S_{j}^{-1}$ is

$$
S_{j}^{-1}\left(r, r^{\prime}\right)=\left\{\begin{array}{l}
\frac{1}{2 \mu_{j}}\left(\frac{r}{r^{\prime}}\right)^{\mu_{j}}\left|\frac{d r}{r} \frac{d r^{\prime}}{r^{\prime}}\right|^{1 / 2}, \quad r<r^{\prime} \\
\frac{1}{2 \mu_{j}}\left(\frac{r^{\prime}}{r}\right)^{\mu_{j}}\left|\frac{d r}{r} \frac{d r^{\prime}}{r^{\prime}}\right|^{1 / 2}, \quad r>r^{\prime} .
\end{array}\right.
$$

Hence

$$
\left(I_{b}(\tilde{P})\right)^{-1}\left(s, y, y^{\prime}\right)= \begin{cases}\frac{1}{2} \sum_{j} \frac{1}{\mu_{j}} u_{j}(y) \overline{u_{j}\left(y^{\prime}\right)} s^{+\mu_{j}}\left|\frac{d s}{s} d h d h^{\prime}\right|^{1 / 2}, \quad s>1, \\ \frac{1}{2} \sum_{j} \frac{1}{\mu_{j}} u_{j}(y) \overline{u_{j}\left(y^{\prime}\right)} s^{-\mu_{j}}\left|\frac{d s}{s} d h d h^{\prime}\right|^{1 / 2}, \quad s<1,\end{cases}
$$

with $s=r / r^{\prime}$. The convergence of this sum can be analyzed using Lemma 4.4; the sum converges smoothly for $s<1$ and for $s>1$. 
Now we determine the leading behaviour of $\tilde{G}$ at zf. We only consider the case $r / r^{\prime}<1 / 4$, as the case $r / r^{\prime}>4$ is completely parallel. Recall from expression (4.11) that for $r / r^{\prime}<1 / 4$ we have

$$
\tilde{G}\left(r, r^{\prime}, y, y^{\prime}\right)=\sum_{j} u_{j}(y) \overline{u_{j}\left(y^{\prime}\right)} I_{\mu_{j}}(r) K_{\mu_{j}}\left(r^{\prime}\right)\left|\frac{d r}{r} \frac{d r^{\prime}}{r^{\prime}} d h d h^{\prime}\right|^{1 / 2} .
$$

We use the limiting forms for small arguments from Section 9.6 of [1]: when $r, r^{\prime} \rightarrow 0$

$$
I_{\mu_{j}}(r)=\frac{r^{\mu_{j}}}{2^{\mu_{j}} \Gamma\left(\mu_{j}+1\right)}\left(1+O\left(r^{2}\right)\right)
$$

and

$$
K_{\mu_{j}}\left(r^{\prime}\right)=\frac{2^{\mu_{j}-1} \Gamma\left(\mu_{j}\right)}{r^{\prime \mu_{j}}}\left(1+O\left(r^{\epsilon}\right)\right)
$$

where we can take $\epsilon$ to be $\min \left(2,2 \mu_{0}\right)$ if $\mu_{0} \neq 1$, or any number less than 2 if $\mu_{0}=1$ (since then there is a $\log$ term $r^{\prime} \log r^{\prime}$ in the expansion of $K_{1}\left(r^{\prime}\right)$ ). By Proposition 4.3 the error terms are uniform in $\mu_{j}$, so we can sum over $j$ to obtain

$$
\tilde{G}_{0}\left(r, r^{\prime}, y, y^{\prime}\right)=\frac{1}{2} \sum_{j} \frac{1}{\mu_{j}} u_{j}(y) \overline{u_{j}\left(y^{\prime}\right)}\left(\frac{r}{r^{\prime}}\right)^{\mu_{j}}\left|\frac{d r}{r} \frac{d r^{\prime}}{r^{\prime}} d h d h^{\prime}\right|^{1 / 2}+O\left(r^{\epsilon}\right) .
$$

Since $d r / r d r^{\prime} / r^{\prime}=d s / s d r^{\prime} / r^{\prime}$, the expression (4.27) shows that the restriction of $\tilde{G}\left(r, r^{\prime}, y, y^{\prime}\right)$ to zf is identical to the restriction $I_{b}\left(\tilde{G}_{n d}^{z f}\right)$ of $\tilde{G}_{n d}^{z f}$ to zf. Since the cutoff function used to define $G_{f}$ is $\chi\left(4 r / r^{\prime}\right)+\chi\left(4 r^{\prime} / r\right)$, and that used to define $G_{n d}$ is $1-\chi\left(4 r / r^{\prime}\right)-\chi\left(4 r^{\prime} / r\right)$, we see that $G_{a}$ has the same leading asymptotic at zf as $G_{n d}^{z f}=r r^{\prime} \tilde{G}_{n d}^{z f}$, namely $r r^{\prime} I_{b}(\tilde{P})^{-1}$.

\subsection{Construction of $P^{-1}$}

We have constructed an approximate inverse $G_{a}=G_{f}+G_{n d}$; let $E$ be the corresponding error term:

$$
P G_{a}=\mathrm{Id}+E \text {. }
$$

We next try to solve away $E$ to obtain our final $G=P^{-1}$. We begin by summarising the properties of $G_{a}$ and $E$.

Proposition 4.6. As a multiple of the Riemannian half-density $\mid r^{d-1} r^{\prime d-1} d r d r^{\prime} d h$ $\left.d h^{\prime}\right|^{1 / 2}$ on $M_{b, \mathrm{sc}}^{2}$, the kernel $G_{a}$ is the sum of two terms. One is $G_{n d}$, supported where $1 / 8 \leq r / r^{\prime} \leq 8$, and is such that $\rho_{\mathrm{zf}}^{d-2} G_{n d}$ is conormal of order -2 with respect to the diagonal uniformly up to both $\mathrm{zf}$ and $\mathrm{sf}$, where $\rho_{\mathrm{zf}}$ is any boundary defining function for $\mathrm{zf}$, and is rapidly decreasing at bf. The other term $G_{f}=$ $G_{a}-G_{n d}$ satisfies:

(i) it is smooth at the diagonal, and polyhomogeneous conormal at all boundary hypersurfaces; 
(ii) it vanishes to infinite order at $\mathrm{lbi}, \mathrm{rbi}$, and $\mathrm{bf}$;

(iii) it vanishes to order $1-d / 2+\mu_{0}$ at $\mathrm{lbz}$ and $\mathrm{rbz}$;

(iv) it vanishes to order $2-d$ at $\mathrm{zf}$.

Proof. The properties of $G_{n d}$ follow from properties of the full $b$-calculus and of the scattering calculus recalled in Section 2.

The diagonal part of property (i) of $G_{f}$ is clear; in fact, it is supported away from the diagonal. Polyhomogeneity of $G_{f}$ at $\mathrm{lbz}$ and $\mathrm{rbz}$ follows from Proposition 4.5 and the symmetry of $G_{f}$, while polyhomogeneity (in a trivial sense, with an empty index set) at lbi, rbi, and bf follows from the exponential decrease of $G_{f}$ as $r$ or $r^{\prime}$ tends to infinity, as shown by Lemma 4.4 .

We obtain the vanishing order at lbz from equations (4.11), (4.18), and (4.19). Since $r$ is the boundary defining function for lbz, we need to compute its power. Clearly one power of $r$ comes from (4.19), while $I_{\mu_{0}}(r)$ in (4.11) gives us the power $r^{\mu_{0}}$. Then the difference between the $b$-half density and the Riemannian half-density gives us a power of $r^{-d / 2}$ (as in (4.7)). Combining these we conclude that the vanishing order at $\mathrm{lbz}$ is $1-d / 2+\mu_{0}$. The vanishing order at $\mathrm{rbz}$ is similar.

Last, we show (iv). Since both $r$ and $r^{\prime}$ vanish at $z f$, to obtain the vanishing order of $G_{a}$ at $z f$, as a scattering-half-density, we combine the powers of $r$ and $r^{\prime}$ in (4.21) and (4.19) with the factor $\left(r r^{\prime}\right)^{-d / 2}$ involved in the change from a $b$-half density to the Riemannian half-density. So the order of vanishing is $1-d / 2+1-$ $d / 2=2-d$.

Proposition 4.7. The error term $E$ has the following properties on $M_{b, \mathrm{sc}}^{2}$ :

(i) it is smooth in the interior;

(ii) it vanishes to first order (as a b-half-density, or to order 1 - $d$ as a Riemannian half-density) at the $z f$-face;

(iii) it vanishes to infinite order at $\mathrm{lbz}, \mathrm{rbz}, \mathrm{lbi}, \mathrm{rbi}, \mathrm{sf}$, and $\mathrm{bf}$;

(iv) it is compact on $L^{2}(M)$; in fact its Schwartz kernel is Hilbert-Schmidt.

Moreover, the $k$-fold composition $E^{k}$ satisfies similar conditions, with (ii) strengthened to vanishing to order $k$ at $\mathrm{zf}$ as a b-half-density.

Proof. Property (i) follows from the choice of $G_{n d}$. Property (ii) follows from the fact that the indicial operator of $\left(r r^{\prime}\right)^{-1} G_{a}$ is equal to $I_{b}(\tilde{P})^{-1}$, as shown in the previous subsection. To show property (iii), consider the $G_{f}$ term. Since $G_{f}$ is equal to the exact inverse of $P$ outside the region $\left\{1 / 8 \leq r / r^{\prime} \leq 8\right\}, P G_{f}$ is supported in this region, hence vanishes in a neighbourhood of lbz, rbz, lbi, and rbi (see Figure 2). On the other hand, by Proposition 4.5, $G_{f}$ has empty index set at bf and sf, so $P G_{f}$ vanishes to all orders there. On the other hand, by (4.22), $G_{n d}$ contributes an error term $E_{n d}$ that is smooth across the diagonal and vanishes to infinite order at all boundary hypersurfaces other than zf. This establishes (iii). Properties (i), (ii), and (iii) show that $E$ has an $L^{2}$ kernel, proving property (iv).

To show the last remark, we use a smooth cutoff function to divide $E$ into two parts, $E=E_{b}+E_{\mathrm{sc}}$, where $E_{b}$ is an order $-\infty$ operator in the $b$-calculus, vanishing 
to first order at zf, and $E_{\mathrm{sc}}$ is an order $(-\infty, \infty)$ operator in the scattering calculus. Then $E^{k}=\left(E_{b}+E_{\mathrm{sc}}\right)^{k}$. Any mixed terms will vanish to infinite order at each boundary hypersurface. Of the remaining terms, using the composition properties of the $b$-calculus and scattering calculus recalled in Section $2, E_{b}^{k}$ is order $-\infty$ in the $b$-calculus and vanishes to order $k$ at $\mathrm{zf}$, while $E_{\mathrm{sc}}^{k}$ is order $(-\infty, \infty)$ in the scattering calculus. Moreover, $E^{k}$ is supported where $\left\{8^{-k} \leq r / r^{\prime} \leq 8^{k}\right\}$, hence vanishes in a neighbourhood of lbz, rbz, lbi, and rbi.

We proceed to solve away $E$. To do so, we would like to invert $\mathrm{Id}+E$. However, it might not be invertible; if not, we perturb $G_{a}$ so that Id $+E$ becomes invertible.

To do this, we first observe that since $E$ is compact on $L^{2}(M)$, according to Proposition 4.7, Id $+E$ is Fredholm of index 0 , and its null space and cokernel both have the same finite dimension, say $N$. Removing the null space gives us an invertible operator, and to achieve that we add a rank $N$ operator to $G_{a}$. To construct the rank $N$ operator we need the following lemma.

Lemma 4.8. Let $\mathcal{S} \subset L^{2}(M)$ be the set of functions

$$
\begin{aligned}
\mathcal{S}=\left\{g \in C^{\infty}(M):\right. & \nabla^{(k)} g=O\left(r^{\infty}\right) \text { as } r \rightarrow 0 \text { and } \\
& \left.O\left(r^{-\infty}\right) \text { as } r \rightarrow \infty, \text { for all } k \in \mathbb{N}_{0}\right\} .
\end{aligned}
$$

There exist smooth functions $\psi_{1}, \ldots, \psi_{N}, \phi_{1}, \ldots, \phi_{N} \in \mathcal{S}$ such that

(i) $\psi_{1}, \ldots, \psi_{N}$ span the null space of $\mathrm{Id}+E$, and

(ii) $P \phi_{1}, \ldots, P \phi_{N}$ span a space complementary to the range of $\mathrm{Id}+E$.

Proof. We choose the $\psi_{i}$ to be any basis of the null space of $\mathrm{Id}+E$. To show $\psi_{i} \in \mathcal{S}$, we note that $\psi_{i}=-E\left(\psi_{i}\right)$, hence iterating, we have $\psi_{i}=E^{2 N} \psi_{i}$ for each $N \geq 1$. Now we consider mapping properties of the operator $E^{N}$. First, writing $E=E_{b}+E_{\mathrm{sc}}$ as in the proof of Proposition 4.7, it is easy to see that $E_{\mathrm{sc}}$ and $\nabla E_{\mathrm{sc}} \operatorname{map} L^{2}(M)$ to $\langle r\rangle^{-L} L^{2}(M)$ for arbitrary $L$. (Here $\nabla$ is shorthand for the vector of derivatives $\left(\partial_{r}, r^{-1} \partial_{y_{i}}\right)$.) As for $E_{b}$, since it has negative order in the $b$ calculus and vanishes to first order at zf, we see that $E_{b}$ maps $L^{2}(M)$ to $r L^{2}(M)$. Since the kernel $\left(r / r^{\prime}\right)^{a} E$ has the same properties as $E$ listed in Proposition 4.7, it follows that $E_{b}$ maps $r^{a} L^{2}(M)$ to $r^{a+1} L^{2}(M)$ for any $a$. Also, applying a derivative $\nabla_{z}=\left(\partial_{r}, r^{-1} \partial_{y_{i}}\right)$ or $\nabla_{z^{\prime}}=\left(\partial_{r^{\prime}}, r^{\prime-1} \partial_{y_{i}^{\prime}}\right)$ to $E_{b}$, it is still of negative order in the $b$-calculus, though no longer vanishing at zf, so we see that both $\nabla_{z} E_{b}$ and $\nabla_{z^{\prime}} E_{b}$ map $r^{a} L^{2}(M)$ to $r^{a} L^{2}(M)$ for any $a$. Summarizing, we have

$$
\begin{aligned}
& E \text { acts as a bounded map } r^{a} L^{2}(M) \rightarrow r^{a+1}\langle r\rangle^{-L} L^{2}(M), \\
& \nabla_{z} E \text { and } \nabla_{z^{\prime}} E \text { act as bounded maps } r^{a} L^{2}(M) \rightarrow r^{a}\langle r\rangle^{-L} L^{2}(M) .
\end{aligned}
$$

Applying these properties of $E$ iteratively, we see that

(4.29) $\nabla^{\left(N_{1}\right)}\left(r^{-N}\langle r\rangle^{2 N} \nabla^{\left(N-N_{1}\right)} E^{2 N}\right)$ acts as a bounded map $L^{2}(M)$ to $L^{2}(M)$

for any $N$ and $0 \leq N_{1} \leq N$. 
In (4.29) the derivatives may be either in the left variable $z$ or the right variable $z^{\prime}$. Hence, considering left derivatives, taking $N_{1}>d / 2$ and using the Sobolev embedding $H^{N_{1}}(M) \rightarrow L^{\infty}(M)$ shows that $\psi_{i}$ is in $r^{N}\langle r\rangle^{-2 N} C^{N-N_{1}}(M)$. Letting $N \rightarrow \infty$, we see that $\psi_{i}$ is smooth and its derivatives have rapid decay both as $r \rightarrow 0$ and $r \rightarrow \infty$, hence $\psi_{i} \in \mathcal{S}$.

As for the $\phi_{i}$, to show that we can choose functions $\phi_{1}, \ldots, \phi_{N}$ as above, let $J$ be the closed subspace of $L^{2}(M)$ given by the closure of the range of $P$ restricted to the subspace $\mathcal{S}$. It suffices to show that $J$ contains a subspace complementary to the range of $\mathrm{Id}+E$, as if that is true, then we can choose a basis for such a subspace, approximate each with points of the form $P \phi_{i}, \phi_{i} \in \mathcal{S}$, and then the span of the $P \phi_{i}$ will also be a complementary subspace, provided the approximations are sufficiently good.

We claim that the orthocomplement of $J$ is a finite dimensional subspace given by

$$
K=\left\{f \in L^{2}(M):\left(\Delta+V_{0} / r^{2}+1\right) f(r, y)=0 \text { for } r>0\right\} .
$$

Notice that then $f$ cannot be in the domain of $P$ (unless $f=0$ ), since $H$ is a nonnegative operator, so $P=H+1$ has trivial null space. Nevertheless it is possible that $K$ is nontrivial; to give an example, if $M=\mathbb{R}^{3}$ and $V_{0}=0$ then $K$ would be one-dimensional, spanned by the function $e^{-r} / r$. In general, $K$ is spanned by functions of the form $r^{-(d-2) / 2} K_{\mu_{j}}(r) u_{j}(y)$ with $\mu_{j}<1$; it is straightforward to check that these are $O\left(r^{-d / 2+1-\mu_{j}}\right)$ as $r \rightarrow 0$, and exponentially decreasing as $r \rightarrow \infty$, hence in $L^{2}(M)$.

To show that $K$ is indeed the orthocomplement of $J$, let $f \in J^{\perp}$. Then

$$
\langle P u, f\rangle=0, \quad \text { for all } u \in \mathcal{S} .
$$

In particular, this is true for $u \in \mathcal{S}$ supported away from $r=0$. This implies that $P f=0$ distributionally away from $r=0$. By elliptic regularity this means that $f$ is smooth for $r \neq 0$. Then we can integrate by parts and deduce

$$
\left\langle u,\left(\Delta+V_{0} / r^{2}+1\right) f\right\rangle=0, \quad \text { for all } u \in \mathcal{S} \text { supported away from } r=0 .
$$

By density of this space of functions $u$, we see that $\left(\Delta+V_{0} / r^{2}+1\right) f(r, y)=0$ a.e. Since $f$ is smooth for $r>0$ this means that $\left(\Delta+V_{0} / r^{2}+1\right) f(r, y)=0$ for $r>0$, i.e, $f \in K$. Conversely, if $f \in K$ then $f$ is orthogonal to $J$, since integration by parts is justified for $u \in \mathcal{S}$.

To complete the proof it remains to show that $J$ contains a subspace complementary to the range of $\mathrm{Id}+E$. This is equivalent to the condition that

$$
J+\operatorname{ran}(\operatorname{Id}+E)=L^{2}(M) .
$$

In turn, this is equivalent to

$$
J^{\perp} \cap(\operatorname{ran}(\mathrm{Id}+E))^{\perp}=\{0\} .
$$

Using $J^{\perp}=K$, this is equivalent to

$$
K \cap \operatorname{null}\left(\operatorname{Id}+E^{*}\right)=\{0\} .
$$


The last condition is straightforward to verify. In fact, $E^{*}$ also satisfies properties (i)-(iv) of Proposition 4.7, so just as above we see that null( $\left.\mathrm{Id}+E^{*}\right)$ is contained in $\mathcal{S}$. Certainly $\mathcal{S}$ is contained in $\operatorname{dom} P$. However, there are no nontrivial solutions to $P f=0$ for $f \in \operatorname{dom} P$, since $P=H+1$ and $H$ is a positive operator. Hence (4.30) is satisfied, which completes the proof.

Let $Q$ be the rank $N$ operator

$$
Q=\sum_{i=1}^{N} \phi_{i}\left\langle\psi_{i}, \cdot\right\rangle,
$$

where $\left\langle\psi_{i}, \cdot\right\rangle$ means the inner product with $\psi_{i}$. The functions $\psi_{1}, \ldots, \psi_{N}, \phi_{1}, \ldots \phi_{N}$ are chosen as in Lemma 4.8. Then we have

$$
P\left(G_{a}+Q\right)=\mathrm{Id}+E+P Q
$$

which is invertible. From this we obtain

$$
P^{-1}=\left(G_{a}+Q\right)(\mathrm{Id}+E+P Q)^{-1} .
$$

Using property (ii) of Lemma 4.8, we see that $G_{a}+Q$ has the 'same' properties as $G_{a}$, i.e., it has those properties listed in Proposition 4.6, and $E^{\prime}:=E+P Q$ has properties (i)-(iv) listed in Proposition 4.7. Define an operator $S$ by

$$
S=\left(\mathrm{Id}+E^{\prime}\right)^{-1}-\mathrm{Id} .
$$

Then we can write

$$
P^{-1}=\left(G_{a}+Q\right)(\mathrm{Id}+S) .
$$

We need to know the properties of $S$.

Lemma 4.9. The operator $S$ has properties (i)-(iv) listed in Proposition 4.7.

Remark 4.10. A similar analysis was made in Section 5.4 of [29].

Proof. Using the identities $(\operatorname{Id}+S)\left(\operatorname{Id}+E^{\prime}\right)=\left(\operatorname{Id}+E^{\prime}\right)(\operatorname{Id}+S)=\operatorname{Id}$, we obtain

$$
S=-E^{\prime}+E^{\prime 2}+E^{\prime} S E^{\prime} .
$$

For any positive integer $N$, we substitute the expression (4.31) into itself $2 N-1$ times, and we get

$$
S=\sum_{j=1}^{4 N}(-1)^{j} E^{\prime j}+E^{\prime 2 N} S E^{2 N}
$$

Using the last part of Proposition 4.7, we see that the term $\sum_{j=1}^{4 N}(-1)^{j} E^{\prime j}$ has all the properties listed in the Lemma, so we focus on the term $S_{N}:=E^{\prime 2 N} S E^{\prime 2 N}$. Using (4.29), we see that

$$
\nabla_{z}^{\left(N_{1}\right)}\left(r^{-N}\langle r\rangle^{2 N} \nabla_{z}^{\left(N-N_{1}\right)} E^{\prime 2 N}\right) \quad \text { and } \quad \nabla_{z^{\prime}}^{\left(N_{1}\right)}\left(r^{\prime-N}\left\langle r^{\prime}\right\rangle^{2 N} \nabla_{z^{\prime}}^{\left(N-N_{1}\right)} E^{\prime 2 N}\right)
$$


are bounded operators on $L^{2}$. Since $S$ is Hilbert-Schmidt, it follows that

$$
\begin{aligned}
& \nabla_{z}^{\left(N_{1}\right)} \nabla_{z^{\prime}}^{\left(N_{1}\right)}\left(\left(r r^{\prime}\right)^{-N}\langle r\rangle^{2 N}\left\langle r^{\prime}\right\rangle^{2 N} \nabla_{z}^{\left(N-N_{1}\right)} \nabla_{z^{\prime}}^{\left(N-N_{1}\right)} S_{N}\right) \\
& \quad=\nabla_{z}^{\left(N_{1}\right)}\left(r^{-N}\langle r\rangle^{2 N} \nabla_{z}^{\left(N-N_{1}\right)} E^{\prime 2 N}\right) \circ S \circ \nabla_{z^{\prime}}^{\left(N_{1}\right)}\left(r^{\prime-N}\left\langle r^{\prime}\right\rangle^{2 N} \nabla_{z^{\prime}}^{\left(N-N_{1}\right)} E^{\prime 2 N}\right)
\end{aligned}
$$

is also Hilbert-Schmidt, i.e., has an $L^{2}$ kernel. Using Sobolev embeddings as in the argument below (4.29), this gives regularity and vanishing (at the boundary of $M_{b, \mathrm{sc}}^{2}$ ) of $S_{N}$ of some finite order $N-O(1)$, and hence the same finite order regularity and vanishing of $S$. Since this argument can be made for any $N$, this proves that $S$ has the properties (i)-(iv) listed in Proposition 4.7.

To summarise, we have

$$
G=P^{-1}=\left(G_{a}+Q\right)(\operatorname{Id}+S),
$$

where $G_{a}+Q$ has those properties listed in Proposition 4.6, Id $+S$ is a compact operator, and $S$ has those properties listed in Lemma 4.9. Our final step is to analyze the composition $\left(G_{a}+Q\right)(\mathrm{Id}+S)$ and show that $G$ itself satisfies all the conditions listed in Proposition 4.6. We summarise key information about $G=P^{-1}$ obtained through our construction in the following theorem. To state it, define $\omega=1-\chi\left(4 r / r^{\prime}\right)-\chi\left(4 r^{\prime} / r\right)$ where $\chi$ is as in (4.18); thus, $\omega$ is a smooth function on the blown-up space supported away from lbz, lbi, rbz, and rbi, and equal to 1 on a neighbourhood of the diagonal. Also let $\rho_{\text {zf }}$ be a boundary defining function for zf.

Theorem 4.11. Let $G_{c}=\omega G$ and $G_{s}=(1-\omega) G$. Then, as a multiple of the Riemannian half-density, i.e., the scattering-half-density $\left|r^{d-1} r^{\prime d-1} d r d r^{\prime} d h d h^{\prime}\right|^{1 / 2}$, on $M_{b, \mathrm{sc}}^{2}, \rho_{\mathrm{zf}}^{d-2} G_{c}$ is conormal of order -2 with respect to the diagonal uniformly up to both $z f$ and sf, while $G_{s}$ satisfies properties (i)-(iv) listed in Proposition 4.6.

Remark 4.12. The subscripts $c$ and $s$ are chosen to indicate that $G_{c}$ is the part of $G$ which is conormal at the diagonal, while $G_{s}$ is the part of $G$ which is smooth at the diagonal.

Proof. We have already proved these properties for $G_{a}$, in Proposition 4.6, so we need to check them for the terms $Q+Q S+G_{a} S=G-G_{a}$. Since $Q$ and $Q S$ both are smooth and vanish to infinite order at the boundaries, these terms trivially satisfy all the conditions. So it remains to check that $G_{a} S$ has the same properties as $G_{a}$.

We write $G_{a} S$ as a sum of two parts. Let $\eta:[0, \infty) \rightarrow[0,1]$ be a smooth cutoff function such that $\eta([0,1])=0$ and $\eta([2, \infty))=1$. The first part $\eta(r) G_{a} \eta\left(r^{\prime}\right)$ is in the scattering calculus. Note that $\eta\left(2 r^{\prime}\right) S$ is also in the scattering calculus, and that

$$
\left(\eta(r) G_{a} \eta\left(r^{\prime}\right)\right)\left(\eta\left(2 r^{\prime}\right) S\right)=\left(\eta(r) G_{a} \eta\left(r^{\prime}\right)\right) S .
$$

Therefore by Proposition 2.17, this term is in the scattering calculus. The second part $G_{a}-\eta(r) G_{a} \eta\left(r^{\prime}\right)$ is in the full $b$-calculus. (Although the support of this term meets the boundary hypersurfaces lbi and rbi, its Schwartz kernel is rapidly vanishing there, enabling us to regard it as living in the $b$-calculus.) In a similar 
sense, $S$ is in the small $b$-calculus (it vanishes rapidly at every boundary hypersurface except zf). Therefore by Proposition 2.12, $\left(G_{a}-\eta(r) G_{a} \eta\left(r^{\prime}\right)\right) S$ is in the full $b$-calculus, with the same index sets at $\mathrm{lbz}$ and $\mathrm{rbz}$ as $G_{a}$. Therefore the required properties for $G_{c}$ and property (i) for $G_{s}$ follow. Also, since $S$ vanishes to first order at zf, the same is true for the composition $\left(G_{a}-\eta(r) G_{a} \eta\left(r^{\prime}\right)\right) S$. So $G_{a} S$ has the same vanishing orders (or better) at the boundary hypersurfaces as $G_{a}$.

Remark 4.13. In the case of the potential $V \equiv 0$, we have $\mu_{0}=d / 2-1$. So the vanishing order in item (iii) of Theorem 4.11 becomes 0 . This is consistent with the case when the cone is $\mathbb{R}^{d}$ and the potential $V \equiv 0$, when the cone tip can be chosen arbitrarily, and $G$ is smooth everywhere.

The vanishing orders of $G=P^{-1}$ at various boundaries of $M_{b, \mathrm{sc}}^{2}$ are shown in Figure 3 .

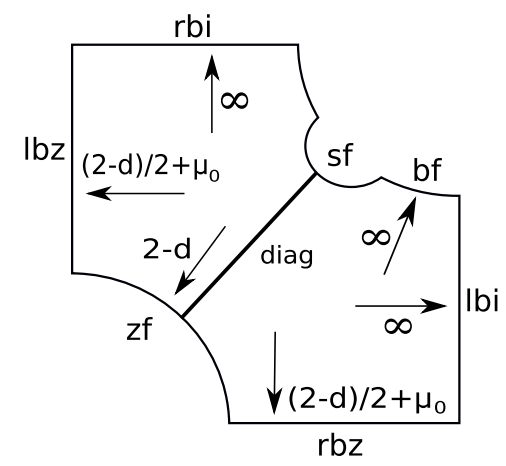

FiguRE 3. The vanishing properties at various boundaries

Remark 4.14. The construction of $G=P^{-1}$ in this section is sketched in p. $885 \mathrm{ff}$ of [27] by C. Guillarmou and the first author, but details are lacking. It is not fully justified in [27] that the kernel is in the scattering calculus near sf, and in the $b$-calculus near zf. For this reason we have given complete details in this section.

\section{The boundedness of the Riesz transform}

\subsection{Estimate on the kernel}

Recall that the Riesz transform $T$ with the inverse square potential $V=V_{0} / r^{2}$, defined in Section 4, can be expressed as

$$
T=\frac{2}{\pi} \int_{0}^{\infty} \nabla\left(H+\lambda^{2}\right)^{-1} d \lambda,
$$

where $H$ is given by (1.4), and recall that $H$ is homogenous of degree -2 . Our analysis of the Riesz transform will be based on the following estimate on the kernel $T\left(z, z^{\prime}\right)$. 
Proposition 5.1. We have the following estimate on the kernel of $T$ :

$$
\left|T\left(z, z^{\prime}\right)\right| \lesssim \int_{0}^{\infty} \lambda^{d-2}\left|\nabla\left(G\left(\lambda z, \lambda z^{\prime}\right)\right)\right| d \lambda,
$$

where $G=P^{-1}=(H+1)^{-1}$, with the properties listed in Theorem 4.11.

Proof. This comes from the relationship between $\left(H+\lambda^{2}\right)^{-1}$ and $G=(H+1)^{-1}$, which is

$$
\left(H+\lambda^{2}\right)^{-1}\left(z, z^{\prime}\right)=\lambda^{d-2}(H+1)^{-1}\left(\lambda z, \lambda z^{\prime}\right) .
$$

The power -2 of $\lambda$ appears because $H$ is homogenous of degree -2 . Remember these kernels are Riemannian half-densities, and this accounts for the power $d$ of $\lambda$ :

$$
\left|(\lambda r)^{d-1}\left(\lambda r^{\prime}\right)^{d-1} d(\lambda r) d\left(\lambda r^{\prime}\right) d h d h^{\prime}\right|^{1 / 2}=\lambda^{d}\left|r^{d-1} r^{\prime d-1} d r d r^{\prime} d h d h^{\prime}\right|^{1 / 2} .
$$

\subsection{Boundedness on $L^{2}(M)$}

Proposition 5.2. The Riesz transform $T$ with the inverse square potential $V=$ $V_{0} / r^{2}$ is bounded on $L^{2}(M)$.

Proof. Our assumption (1.3) implies that for some small positive $\varepsilon$,

$$
\Delta_{Y}+V_{0}(y)+\left(\frac{d-2}{2}\right)^{2}>\frac{\varepsilon}{1-\varepsilon} V_{0}(y)
$$

Hence $\Delta_{Y}+V_{0}(y) /(1-\epsilon)+((d-2) / 2)^{2}>0$. It follows from Proposition 4.1 that $\Delta+\frac{1}{(1-\varepsilon) r^{2}} V_{0}(y)>0$, or equivalently $\Delta+\frac{1}{r^{2}} V_{0}(y)>\varepsilon \Delta$. From this,

$$
\begin{aligned}
\langle T f, T f\rangle & =\left\langle\Delta\left(\Delta+\frac{1}{r^{2}} V_{0}(y)\right)^{-1 / 2} f,\left(\Delta+\frac{1}{r^{2}} V_{0}(y)\right)^{-1 / 2} f\right\rangle \\
& \leq\left\langle\varepsilon^{-1}\left(\Delta+\frac{1}{r^{2}} V_{0}(y)\right)\left(\Delta+\frac{1}{r^{2}} V_{0}(y)\right)^{-1 / 2} f,\left(\Delta+\frac{1}{r^{2}} V_{0}(y)\right)^{-1 / 2} f\right\rangle \\
& =\varepsilon^{-1}\left\langle\left(\Delta+\frac{1}{r^{2}} V_{0}(y)\right)^{1 / 2} f,\left(\Delta+\frac{1}{r^{2}} V_{0}(y)\right)^{-1 / 2} f\right\rangle=\varepsilon^{-1}\langle f, f\rangle .
\end{aligned}
$$

Therefore $T$ is bounded on $L^{2}(M)$.

\subsection{The diagonal region}

To understand the Riesz transform on $L^{p}$, we decompose $G$ as in Theorem 4.11. Here we will write $G_{1}$ for $G_{c}=\omega G$ (recall $\omega=1-\chi\left(4 r / r^{\prime}\right)-\chi\left(4 r^{\prime} / r\right)$ ), and we further decompose $G_{s}=G_{2}+G_{3}$, where $G_{2}=G \chi\left(4 r / r^{\prime}\right)$ and $G_{3}=G \chi\left(4 r^{\prime} / r\right)$. Thus $G=G_{1}+G_{2}+G_{3}$. Notice that $G_{2}$ and $G_{3}$ are supported away from the diagonal, in particular where the infinite series (4.11) has good convergence properties as shown in Proposition 4.5. We correspondingly decompose the Riesz transform into three pieces. See Figure 4. Thus we have

$$
T_{i}\left(z, z^{\prime}\right)=\frac{2}{\pi} \int_{0}^{\infty} \lambda^{d-2} \nabla_{z}\left(G_{i}\left(\lambda z, \lambda z^{\prime}\right)\right) d \lambda .
$$



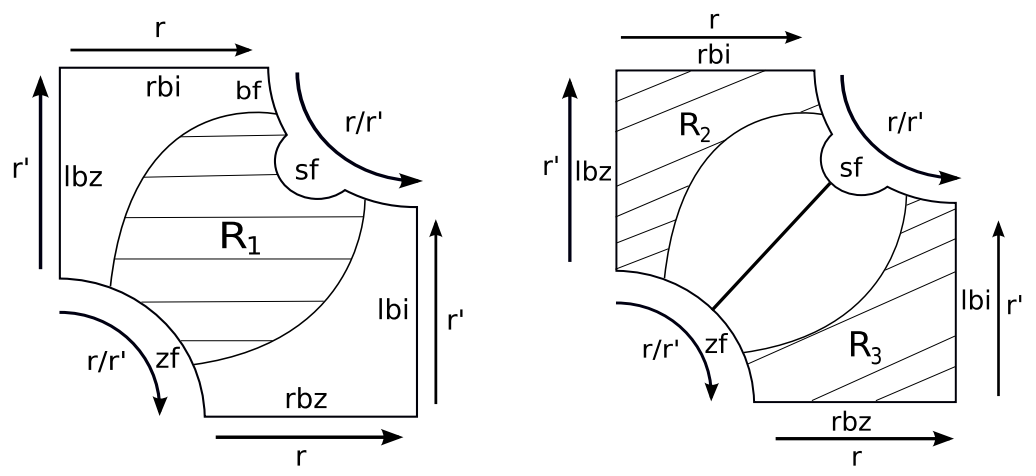

Figure 4. The operators $G_{i}$ and $T_{i}$ are supported in region $R_{i}, i=1,2,3$.

We now show that $T_{1}$ is of weak type $(1,1)$. For that we first need to estimate the derivatives of $G_{1}$.

Lemma 5.3. Let $d\left(z, z^{\prime}\right)$ denote the distance between $z$ and $z^{\prime}$ on $M$. On the support of $\omega$, we have $\rho_{\mathrm{bf}} \lesssim d\left(z, z^{\prime}\right)^{-1}$, where $\rho_{\mathrm{bf}}$ is a boundary defining function for bf.

Proof. Let $z=(r, y)$ and $z^{\prime}=\left(r^{\prime}, y^{\prime}\right)$. Observe from (3.1) that $d\left(z, z^{\prime}\right)$ is bounded above by $r+r^{\prime}$. Therefore in the region $\left\{1 / 8 \leq r / r^{\prime} \leq 8\right\}$ we have

$$
d\left(z, z^{\prime}\right)^{-1} \geq\left(r+r^{\prime}\right)^{-1}=r^{-1}\left(1+\frac{r}{r^{\prime}}\right)^{-1} \geq \frac{1}{9} r^{\prime-1}
$$

As $r^{\prime-1}$ is a boundary defining function for bf on the support of $\omega$, the result follows.

Lemma 5.4. The kernel $G_{1}$ satisfies the estimate that for any integer $j \geq 0$, we have

$$
\left|\nabla_{z, z^{\prime}}^{j} G_{1}\left(z, z^{\prime}\right)\right| \lesssim \begin{cases}d\left(z, z^{\prime}\right)^{2-d-j}, & d\left(z, z^{\prime}\right) \leq 1 \\ d\left(z, z^{\prime}\right)^{-N}, & d\left(z, z^{\prime}\right) \geq 1\end{cases}
$$

for any $N>0$.

Proof. Note that $G_{1}$ is supported in the region $R_{1}=\left\{1 / 8 \leq r / r^{\prime} \leq 8\right\}$. Since $\rho_{\mathrm{zf}}^{d-2} G_{1}$ is conormal of order -2 with respect to the diagonal, by Proposition 3.1 and Lemma 3.2, near the diagonal we have

$$
\left|\rho_{\mathrm{zf}}^{d-2} G_{1}\left(z, z^{\prime}\right)\right| \lesssim a_{\mathrm{diag}}^{(2-d) / 2} .
$$

Near zf, $a_{\text {diag }}$ is comparable to $d\left(z, z^{\prime}\right)^{2} / \rho_{\mathrm{zf}}^{2}$, so it follows that near zf, we have

$$
\left|G_{1}\left(z, z^{\prime}\right)\right| \lesssim d\left(z, z^{\prime}\right)^{2-d} .
$$

Away from zf, $a_{\text {diag }}$ is comparable to $d\left(z, z^{\prime}\right)^{2}$, therefore

$$
\left|G_{1}\left(z, z^{\prime}\right)\right| \lesssim a_{\mathrm{diag}}^{(2-d) / 2}=d\left(z, z^{\prime}\right)^{2-d}
$$


Now let us consider the behaviour of $G_{1}$ near bf. By Theorem 4.11, we know that $G_{1}$ vanishes to infinite order at bf, while by Lemma 5.3 , we know that $\rho_{\text {bf }} \lesssim$ $d\left(z, z^{\prime}\right)^{-1}$. Therefore near the bf-face we know that

$$
\left|G_{1}\left(z, z^{\prime}\right)\right| \lesssim d\left(z, z^{\prime}\right)^{-N}
$$

for any $N>0$. As for the rest of $R_{1}$, after we take away the neighbourhoods near zf, bf, and the diagonal, we are left with a compact set, on which both $G_{1}$ and $d\left(z, z^{\prime}\right)^{-1}$ are continuous with $d\left(z, z^{\prime}\right)^{-1}$ being nonzero. Therefore we can conclude that

$$
\left|G_{1}\left(z, z^{\prime}\right)\right| \lesssim \begin{cases}d\left(z, z^{\prime}\right)^{2-d}, & d\left(z, z^{\prime}\right) \leq 1 \\ d\left(z, z^{\prime}\right)^{-N}, & d\left(z, z^{\prime}\right) \geq 1\end{cases}
$$

for any $N>0$.

Estimates on the derivatives of $G$ follow in a similar way, using the conormality of $G$. Notice that a derivative $\nabla_{z}$ is of the form $r^{-1}$ times a smooth $b$-derivative for small $r$, and is a smooth scattering vector field for $r$ large. So for $r$ small, for each derivative we lose a factor of $r$ (due to conormality at the boundary) times a factor of $a_{\mathrm{diag}}^{1 / 2}$ (due to conormality at the diagonal) - that is, an overall factor of $d\left(z, z^{\prime}\right)$. Similarly, for $r$ large, for each derivative we lose a factor of $a_{\mathrm{diag}}^{1 / 2}$ due to conormality at the diagonal, which is comparable to $d\left(z, z^{\prime}\right)$ for $r$ large.

Proposition 5.5. The operator $T_{1}$ maps $L^{1}(M)$ into $L^{1, \text { weak }}(M)$.

Proof. We apply Calderón-Zygmund theory; see Section 8.1.1 of [25]. It is sufficient to verify the conditions

(i) $T_{1}$ is bounded on $L^{2}(M)$;

(ii) $\left|T_{1}\left(z, z^{\prime}\right)\right| \leq \frac{C}{\left(d\left(z, z^{\prime}\right)\right)^{d}}$;

(iii) $\left|\nabla_{z} T_{1}\left(z, z^{\prime}\right)\right| \leq \frac{C}{\left(d\left(z, z^{\prime}\right)\right)^{d+1}} \quad$ and $\quad\left|\nabla_{z^{\prime}} T_{1}\left(z, z^{\prime}\right)\right| \leq \frac{C}{\left(d\left(z, z^{\prime}\right)\right)^{d+1}}$,

for some constant $C>0$.

We already know from Proposition 5.2 that $T$ is bounded on $L^{2}(M)$. So to verify condition (i), we just need to show $T-T_{1}$ is bounded on $L^{2}(M)$, which is covered by Proposition 5.13 in Section 5.4.

Now we show conditions (ii) and (iii). By Lemma 5.4 we know the kernel $G_{1}$ satisfies, with any $\lambda>0$,

$$
\left|\nabla_{z}\left(G_{1}\left(\lambda z, \lambda z^{\prime}\right)\right)\right| \leq \lambda\left|\left(\nabla_{z} G_{1}\right)\left(\lambda z, \lambda z^{\prime}\right)\right| \lesssim \begin{cases}\lambda^{2-d} d\left(z, z^{\prime}\right)^{1-d}, & \lambda d\left(z, z^{\prime}\right) \leq 1 \\ \lambda^{-N+1} d\left(z, z^{\prime}\right)^{-N}, & \lambda d\left(z, z^{\prime}\right) \geq 1\end{cases}
$$

and

$$
\left|\nabla_{z}^{2}\left(G_{1}\left(\lambda z, \lambda z^{\prime}\right)\right)\right| \leq \lambda^{2}\left|\left(\nabla_{z}^{2} G_{1}\right)\left(\lambda z, \lambda z^{\prime}\right)\right| \lesssim \begin{cases}\lambda^{2-d} d\left(z, z^{\prime}\right)^{-d}, & \lambda d\left(z, z^{\prime}\right) \leq 1 \\ \lambda^{-N+2} d\left(z, z^{\prime}\right)^{-N}, & \lambda d\left(z, z^{\prime}\right) \geq 1\end{cases}
$$


for any $N>0$. We use this in $(5.1)$ to estimate $T_{1}\left(z, z^{\prime}\right)$ :

$$
\begin{aligned}
\left|T_{1}\left(z, z^{\prime}\right)\right| & \lesssim \int_{0}^{\infty} \lambda^{d-2}\left|\nabla_{z}\left(G_{1}\left(\lambda z, \lambda z^{\prime}\right)\right)\right| d \lambda \\
& \lesssim \int_{0}^{1 / d\left(z, z^{\prime}\right)} d\left(z, z^{\prime}\right)^{1-d} d \lambda+\int_{1 / d\left(z, z^{\prime}\right)}^{\infty} \lambda^{d-N-1} d\left(z, z^{\prime}\right)^{-N} d \lambda \\
& \left.=2 d\left(z, z^{\prime}\right)^{-d} \quad \text { (Choose } N=d+1 .\right)
\end{aligned}
$$

Now estimate the derivative with respect to $z$. The $z^{\prime}$ case is similar.

$$
\begin{aligned}
\left|\nabla_{z} T_{1}\left(z, z^{\prime}\right)\right| & =\frac{2}{\pi}\left|\int_{0}^{\infty} \lambda^{d-2} \nabla_{z} \nabla_{z}\left(G_{1}\left(\lambda z, \lambda z^{\prime}\right)\right) d \lambda\right| \\
& \lesssim \int_{0}^{1 / d\left(z, z^{\prime}\right)} d\left(z, z^{\prime}\right)^{-d} d \lambda+\int_{1 / d\left(z, z^{\prime}\right)}^{\infty} \lambda^{d-N} d\left(z, z^{\prime}\right)^{-N} d \lambda \\
& \left.=2 d\left(z, z^{\prime}\right)^{-d-1} . \quad \text { (Choose } N=d+2 .\right)
\end{aligned}
$$

This completes the proof.

By interpolation, we obtain the following proposition.

Proposition 5.6. The operator $T_{1}$ is bounded on $L^{p}(M)$ for any $p>1$.

Proof. By the Marcinkiewicz interpolation theorem, we know that $T_{1}$ is bounded on $L^{p}(M)$ for all $1<p \leq 2$. Next, because Lemma 5.4 applies to derivatives of $G_{1}$ in both the left and right variables, Proposition 5.5 holds also for the adjoint of $T_{1}$, hence the adjoint is also bounded on $L^{p}(M)$ for all $1<p \leq 2$. Using duality, we get boundedness of $T_{1}$ on $L^{p}(M)$ for $2 \leq p<\infty$.

\subsection{Off-diagonal region}

To study the boundedness of the two off-diagonal operators $T_{2}$ and $T_{3}$, the following lemmas will be useful. They are similar to Lemma 5.4 in [30], but not covered by it.

Lemma 5.7. Consider the kernel $K\left(r, r^{\prime}\right)$ defined by

$$
K\left(r, r^{\prime}\right)= \begin{cases}r^{-\alpha} r^{\prime-\beta}, & r \leq r^{\prime}, \\ 0, & r>r^{\prime} .\end{cases}
$$

If $\alpha+\beta=d, \beta>0$, and $p$ satisfies

$$
p<\frac{d}{\max (\alpha, 0)},
$$

then $K$ is bounded as an operator on $L^{p}\left(\mathbb{R}_{+} ; r^{d-1} d r\right)$.

Proof. The proof is essentially taken from [30]. To find out for what $p$ the operator with kernel $K\left(r, r^{\prime}\right)$ is bounded on $L^{p}\left(\mathbb{R}_{+}, r^{d-1} d r\right)$, we consider the isometry $M: L^{p}\left(\mathbb{R}_{+}, r^{d-1} d r\right) \rightarrow L^{p}\left(\mathbb{R}_{+}, r^{-1} d r\right)$ defined by

$$
(M f)(r)=r^{d / p} f(r) \text {. }
$$


Then the kernel of the operator $\tilde{K}=M K M^{-1}: L^{p}\left(\mathbb{R}_{+}, r^{-1} d r\right) \rightarrow L^{p}\left(\mathbb{R}_{+}, r^{-1} d r\right)$ is

$$
\tilde{K}\left(r, r^{\prime}\right)=r^{d / p} r^{\prime d-d / p} K\left(r, r^{\prime}\right)=\left(\frac{r}{r^{\prime}}\right)^{-\alpha+d / p} \chi_{\left\{r \leq r^{\prime}\right\}} .
$$

Make the substitution $s=\ln r$ and $s^{\prime}=\ln r^{\prime}$. Then $\tilde{K}\left(s, s^{\prime}\right)$ is an operator on $L^{p}(\mathbb{R}, d s)$, and

$$
\tilde{K}\left(s, s^{\prime}\right)=e^{(-\alpha+d / p)\left(s-s^{\prime}\right)} \chi_{\left\{s-s^{\prime} \leq 0\right\}} .
$$

This is a convolution operator, so it is bounded provided the kernel is an $L^{1}$ function with variable $s-s^{\prime}$. Since $s-s^{\prime} \leq 0$, we want $-\alpha+d / p>0$. That is,

$$
p<\frac{d}{\max (\alpha, 0)} .
$$

Since we want $p>1$, we require $\alpha<d$, i.e., $\beta>0$.

Lemma 5.8. Consider the kernel $K\left(r, r^{\prime}\right)$ defined by

$$
K\left(r, r^{\prime}\right)= \begin{cases}0, & r \leq r^{\prime}, \\ r^{-\gamma} r^{\prime-\delta}, & r>r^{\prime} .\end{cases}
$$

If $\gamma+\delta=d, \gamma>0$, and $p$ satisfies

$$
p>\frac{d}{\min (\gamma, d)},
$$

then $K$ is bounded as an operator on $L^{p}\left(\mathbb{R}_{+} ; r^{d-1} d r\right)$.

Proof. By duality and Lemma 5.7.

Corollary 5.9. Let $K\left(r, r^{\prime}, y, y^{\prime}\right)$ be a kernel on the cone $M$ satisfying

$$
\left|K\left(r, r^{\prime}, y, y^{\prime}\right)\right| \leq \begin{cases}r^{-\alpha} r^{\prime-\beta}, & r \leq r^{\prime} \\ 0, & r>r^{\prime}\end{cases}
$$

If $\alpha+\beta=d, \beta>0$, and $p$ satisfies

$$
p<\frac{d}{\max (\alpha, 0)},
$$

then $K$ is bounded as an operator on $L^{p}\left(M ; r^{d-1} d r d h\right)$. Similarly, if $K\left(r, r^{\prime}, y, y^{\prime}\right)$ satisfies

$$
\left|K\left(r, r^{\prime}, y, y^{\prime}\right)\right| \leq \begin{cases}0, & r \leq r^{\prime} \\ r^{-\gamma} r^{\prime-\delta}, & r>r^{\prime}\end{cases}
$$

with $\gamma+\delta=d, \gamma>0$, then if $p$ satisfies

$$
\frac{d}{\min (\gamma, d)}<p
$$

$K$ is bounded as an operator on $L^{p}\left(M ; r^{d-1} d r d h\right)$.

Proof. This follows from Lemmas 5.7 and 5.8 and the fact that the cross section $Y$ has finite volume. 
Proposition 5.10. The operator $T_{2}$ is bounded on $L^{p}(M)$ for

$$
p<\frac{d}{\max \left(d / 2-\mu_{0}, 0\right)},
$$

where $\mu_{0}>0$ is the square root of the smallest eigenvalue of the operator $\Delta_{Y}+$ $V_{0}(y)+((d-2) / 2)^{2}$.

Proof. By Theorem 4.11, we have

$$
\left|G_{2}\left(r, r^{\prime}, y, y^{\prime}\right)\right| \lesssim \rho_{\mathrm{zf}}^{2-d} \rho_{\mathrm{lbz}}^{1-d / 2+\mu_{0}} \rho_{\mathrm{rbi}}^{\infty}=r^{1-d / 2+\mu_{0}} r^{\prime 1-d / 2-\mu_{0}}\left\langle r^{\prime}\right\rangle^{-\infty},
$$

where $\mu_{0}>0$ is as above. On the support of $T_{2}$ and for $r^{\prime} \leq 1$ we can use boundary defining functions

$$
\rho_{\mathrm{zf}}=r^{\prime}, \quad \rho_{\mathrm{lbz}}=\frac{r}{r^{\prime}}, \quad \rho_{\mathrm{rbi}}=1,
$$

while for $r^{\prime} \geq 1$ we can use

$$
\rho_{\mathrm{zf}}=1, \quad \rho_{\mathrm{lbz}}=r, \quad \rho_{\mathrm{rbi}}=r^{\prime-1} .
$$

Therefore, (5.9) implies that

$$
\left|G_{2}\left(\lambda r, \lambda r^{\prime}, y, y^{\prime}\right)\right| \lesssim \begin{cases}\lambda^{2-d} r^{1-d / 2+\mu_{0}} r^{1-d / 2-\mu_{0}}, & \lambda \leq 1 / r^{\prime}, \\ \lambda^{1-d / 2+\mu_{0}-N} r^{1-d / 2+\mu_{0}} r^{\prime-N}, & \lambda \geq 1 / r^{\prime},\end{cases}
$$

for any $N>0$. Then, using the conormality of $G_{2}$ at the boundary, we have

$$
\left|\nabla_{z}\left(G_{2}\left(\lambda r, \lambda r^{\prime}, y, y^{\prime}\right)\right)\right| \lesssim \begin{cases}\lambda^{2-d} r^{-d / 2+\mu_{0}} r^{1-d / 2-\mu_{0}}, & \lambda \leq 1 / r^{\prime} \\ \lambda^{1-d / 2+\mu_{0}-N} r^{-d / 2+\mu_{0}} r^{\prime-N}, & \lambda \geq 1 / r^{\prime}\end{cases}
$$

for all $N>0$, since $\nabla_{z}$ is equal to $1 / r$ times a tangential derivative for small $r$.

Using (5.3) and the fact that $G_{2}$ is supported where $r \leq r^{\prime}$, we estimate

$$
\begin{aligned}
& \left|T_{2}\left(r, r^{\prime}, y, y^{\prime}\right)\right| \lesssim \int_{0}^{\infty} \lambda^{d-2}\left|\nabla_{z}\left(G_{2}\left(\lambda r, \lambda r^{\prime}, y, y^{\prime}\right)\right)\right| d \lambda \\
& \lesssim \int_{0}^{1 / r^{\prime}} \lambda^{d-2}\left(\lambda^{2-d} r^{-d / 2+\mu_{0}} r^{1-d / 2-\mu_{0}}\right) d \lambda \\
& \quad+\int_{1 / r^{\prime}}^{1 / r} \lambda^{d-2}\left(\lambda^{1-d / 2+\mu_{0}-N} r^{-d / 2+\mu_{0}} r^{\prime-N}\right) d \lambda \\
& =r^{-d / 2+\mu_{0}} r^{\prime 1-d / 2-\mu_{0}} \int_{0}^{1 / r^{\prime}} d \lambda+r^{-d / 2+\mu_{0}} r^{\prime-N} \int_{1 / r^{\prime}}^{1 / r} \lambda^{d / 2+\mu_{0}-N-1} d \lambda \\
& =r^{-d / 2+\mu_{0}} r^{\prime-d / 2-\mu_{0}}+\frac{1}{d / 2+\mu_{0}-N}\left(r^{N-d} r^{\prime-N}-r^{-d / 2+\mu_{0}} r^{\prime-d / 2-\mu_{0}}\right) \\
& \lesssim r^{-d / 2+\mu_{0}} r^{\prime-d / 2-\mu_{0}} \quad \text { for } N>\mu_{0}+\frac{d}{2} .
\end{aligned}
$$


By Corollary 5.9, we conclude that $T_{2}$ is bounded on $L^{p}(M)$ provided that

$$
p<\frac{d}{\max \left(d / 2-\mu_{0}, 0\right)} .
$$

Remark 5.11. When $V \equiv 0$, then $\mu_{0}=d / 2-1$, and its first eigenfunction $u_{0}$ is a constant function. In Section 5.5 we will improve estimate (5.10) to obtain a bigger range for $p$ for this special case.

Proposition 5.12. The operator $T_{3}$ is bounded on $L^{p}(M)$ for

$$
p>\frac{d}{\min \left(1+d / 2+\mu_{0}, d\right)},
$$

where $\mu_{0}>0$ is the square root of the smallest eigenvalue of the operator $\Delta_{Y}+$ $V_{0}(y)+((d-2) / 2)^{2}$.

Proof. This proof is very similar to that of Proposition 5.10. By Theorem 4.11, we have

$$
\left|G_{3}\left(r, r^{\prime}, y, y^{\prime}\right)\right| \lesssim \rho_{\mathrm{zf}}^{2-d} \rho_{\mathrm{rbz}}^{1-d / 2+\mu_{0}} \rho_{\mathrm{lbi}}^{\infty}=r^{1-d / 2-\mu_{0}} r^{\prime 1-d / 2+\mu_{0}}\langle r\rangle^{-\infty} .
$$

On the support of $T_{2}$ and for $r \leq 1$ we can use boundary defining functions

$$
\rho_{\mathrm{zf}}=r, \quad \rho_{\mathrm{rbz}}=\frac{r^{\prime}}{r}, \quad \rho_{\mathrm{rbi}}=1,
$$

while for $r \geq 1$ we can use

$$
\rho_{\mathrm{zf}}=1, \quad \rho_{\mathrm{lbz}}=r^{\prime}, \quad \rho_{\mathrm{rbi}}=r^{-1} .
$$

It follows that, as in the proof of Proposition 5.10, by the conormality of $G_{3}$ at the boundary, that for all $N>0$,

$$
\left|\nabla_{z}\left(G_{3}\left(\lambda r, \lambda r^{\prime}, y, y^{\prime}\right)\right)\right| \lesssim \begin{cases}\lambda^{2-d} r^{-d / 2-\mu_{0}} r^{\prime-d / 2+\mu_{0}}, & \lambda \leq 1 / r, \\ \lambda^{-d / 2+\mu_{0}-N+1} r^{-N-1} r^{1-d / 2+\mu_{0}}, & \lambda \geq 1 / r,\end{cases}
$$

Then using (5.3) and the fact that $G_{3}$ is supported where $r^{\prime} \leq r$, we have

$$
\begin{aligned}
& \left|T_{3}\left(r, r^{\prime}, y, y^{\prime}\right)\right| \lesssim \int_{0}^{\infty} \lambda^{d-2}\left|\nabla_{z}\left(G_{3}\left(\lambda r, \lambda r^{\prime}, y, y^{\prime}\right)\right)\right| d \lambda \\
& \lesssim \int_{0}^{1 / r} \lambda^{d-2}\left(\lambda^{2-d} r^{-d / 2-\mu_{0}} r^{\prime 1-d / 2+\mu_{0}}\right) d \lambda \\
& \quad+\int_{1 / r}^{1 / r^{\prime}} \lambda^{d-2}\left(\lambda^{-d / 2+\mu_{0}-N+1} r^{-N-1} r^{1-d / 2+\mu_{0}}\right) d \lambda \\
& =r^{-d / 2-\mu_{0}} r^{\prime 1-d / 2+\mu_{0}} \int_{0}^{1 / r} d \lambda+r^{-N-1} r^{1-d / 2+\mu_{0}} \int_{1 / r}^{1 / r^{\prime}} \lambda^{d / 2+\mu_{0}-N-1} d \lambda \\
& \lesssim r^{-1-d / 2-\mu_{0}} r^{\prime 1-d / 2+\mu_{0}} \text { for } N>\mu_{0}+\frac{d}{2} .
\end{aligned}
$$


Applying Corollary 5.9, we conclude that $T_{3}$ is bounded on $L^{p}(M)$ provided that

$$
p>\frac{d}{\min \left(1+d / 2+\mu_{0}, d\right)} .
$$

Proposition 5.13. The operator $T_{1}$ is bounded on $L^{2}(M)$.

Proof. Since 2 satisfies the boundedness criteria in both Proposition 5.10 and Proposition 5.12, the operator $T_{2}+T_{3}=T-T_{1}$ is bounded on $L^{2}(M)$. The operator $T$ is bounded on $L^{2}(M)$ by Proposition 5.2, and from this the boundedness of $T_{1}$ on $L^{2}(M)$ follows.

Remark 5.14. Proposition 5.13 completes the missing part in the proof of Proposition 5.5.

\subsection{Proofs of main results}

Proof of Theorem 1.3. Since $T=T_{1}+T_{2}+T_{3}$, we just combine Proposition 5.6, Proposition 5.10, and Proposition 5.12 to prove the first part of this theorem.

For the second part, with $V \not \equiv 0$, for $p$ outside the interval (1.8), the counterexamples from Section 5.2 of [27] serve to show the lack of boundedness of $T$ on $L^{p}(M)$. (For purposes of comparison, note that the variables $x$ and $x^{\prime}$ in [27] correspond to $1 / r$ and $1 / r^{\prime}$ in this paper.)

Proof of Theorem 1.1. Suppose that the potential $V$ is identically zero; we proceed to show that the upper threshold for $L^{p}$ boundedness is $p=d\left(d / 2-\mu_{1}\right)^{-1}$. Notice that $T_{1}$ and $T_{3}$ are automatically bounded on this extra range, so we only have to consider $T_{2}$, which has an expression of the form

$$
T_{2}\left(z, z^{\prime}\right)=\frac{2}{\pi} \chi\left(4 r / r^{\prime}\right) \int_{0}^{\infty} \lambda^{d-2} \nabla_{z}\left(G\left(\lambda z, \lambda z^{\prime}\right)\right) d \lambda .
$$

We recall that $G=r r^{\prime} \tilde{G}$ and substitute the infinite series (4.11) for $\tilde{G}$ here, and consider the first term in this sum separately from the rest.

Since $\mu_{0}=d / 2-1$ when $V_{0}=0$, the first term here is

$$
\left(r r^{\prime}\right)^{1-d / 2} u_{0}(y) u_{0}\left(y^{\prime}\right) I_{d / 2-1}(r) K_{d / 2-1}\left(r^{\prime}\right)
$$

times the Riemannian half-density. (Recall this gives us an extra factor of $\left(r r^{\prime}\right)^{-d / 2}$ compared to writing it as a $b$-half-density, as in (4.6) versus (4.7).) When $V_{0}=0$, the eigenfunction $u_{0}(y)$ is constant. Also, $I_{d / 2-1}(r)=c r^{d / 2-1}+O\left(r^{d / 2+1}\right)$ and is conormal at $r=0$, implying that $\nabla_{r}\left(r^{1-d / 2} I_{d / 2-1}(r)\right)=O(r)$. Hence

$$
\nabla_{z}\left(r^{1-d / 2} u_{0}(y) I_{d / 2-1}(r)\right)=O(r)
$$

that is, in this special case, applying the derivative $\nabla_{z}$ makes the kernel vanish to an additional order, instead of one order less as is usually the case. Therefore, after taking the gradient in the left variables, this term is bounded by

$$
C r r^{2-d}, \text { for } r^{\prime} \leq 1, \text { and by } C r r^{\prime-N}, \text { for } r^{\prime} \geq 1 \text {, }
$$


for any integer $N$. Now we substitute this in (5.12) and find that the contribution to $T_{2}$ of the $\mu_{0}$-term is bounded by

$$
\int_{0}^{1 / r^{\prime}} \lambda^{2-d}\left(\lambda^{d} r r^{\prime 2-d}\right) d \lambda+\int_{1 / r^{\prime}}^{\infty} \lambda^{2-d}\left(\lambda^{2-N} r r^{\prime-N}\right) d \lambda \leq C r r^{\prime-1-d} .
$$

Remembering that this term is supported in $\left\{r \leq r^{\prime}\right\}$, we see from Corollary 5.9 that this term is bounded on $L^{p}$ for all $p \in(1, \infty)$.

Consider the remainder of the series. The argument in the previous subsection applies, except that the series now begins with the $\mu_{1}$ term rather than the $\mu_{0}$ term, so we have boundedness in the range (5.8) with $\mu_{1}$ replacing $\mu_{0}$, completing the proof.

\section{A. Estimates on modified Bessel functions}

Proof of Proposition 4.3. From Section 9.6 of [1], we have representations

$$
I_{\mu}(r)=\frac{2^{-\mu} r^{\mu}}{\pi^{1 / 2} \Gamma(\mu+1 / 2)} \int_{-1}^{1}\left(1-t^{2}\right)^{\mu-1 / 2} e^{-r t} d t
$$

and

$$
K_{\mu}(r)=\frac{\pi^{1 / 2} 2^{-\mu} r^{\mu}}{\Gamma(\mu+1 / 2)} \int_{1}^{\infty} e^{-r t}\left(t^{2}-1\right)^{\mu-1 / 2} d t .
$$

By introducing a new integration variable $r \sqrt{t^{2}-1}$ we can convert this to

$$
K_{\mu}(r)=\frac{\pi^{1 / 2} 2^{-\mu} r^{-\mu}}{\Gamma(\mu+1 / 2)} \int_{0}^{\infty} \frac{e^{-\sqrt{r^{2}+t^{2}}}}{\sqrt{r^{2}+t^{2}}} t^{2 \mu} d t
$$

We now estimate each of these integrals in a way that is uniform as $\mu \rightarrow \infty$. It is straightforward to estimate $I_{\mu}$ :

$$
\begin{aligned}
e^{-r} I_{\mu}(r) & =\frac{2^{-\mu} r^{\mu}}{\pi^{1 / 2} \Gamma(\mu+1 / 2)} \int_{-1}^{1}\left(1-t^{2}\right)^{\mu-1 / 2} e^{-r(t+1)} d t \\
& \leq \frac{2^{-\mu} r^{\mu}}{\pi^{1 / 2} \Gamma(\mu+1 / 2)} \int_{-1}^{1} e^{-r(t+1)} d t \leq \frac{2 \cdot 2^{-\mu} r^{\mu}}{\pi^{1 / 2} \Gamma(\mu+1 / 2)}
\end{aligned}
$$

We next estimate $K_{\mu}$. We use

$$
\left|\frac{e^{-\sqrt{r^{2}+t^{2}}}}{\sqrt{r^{2}+t^{2}}}\right| \leq \frac{e^{-r / 2} e^{-t / 2}}{t}
$$

to get

$$
\left|K_{\mu}(r)\right| \leq \pi^{1 / 2} \frac{e^{-r / 2} r^{-\mu} 2^{\mu} \Gamma(2 \mu)}{\Gamma(\mu+1 / 2)}
$$


We also want estimates for the derivatives of $I_{\mu}$ and $K_{\mu}$. For the derivatives of $I_{\mu}$, derivatives in $r$ hit either the $r^{\mu}$ factor outside the integral in (A.2) or the $e^{-r t}$ factor inside the integral. Suppose that $j$ derivatives hit the $r^{-\mu}$ factor and the remainder act inside the integral. The former gives a factor $(-\mu)(-\mu-1)$ $\cdots(-\mu-j+1) r^{-j}$, while the latter give a factor $(-t)^{l-j}$ which is bounded by 1 in magnitude. Thus we get an estimate

$$
\left|\left(\frac{d}{d r}\right)^{l} I_{\mu}(r)\right| \leq C_{l} \mu^{l} \frac{2^{-\mu} r^{\mu-l} e^{r}}{\Gamma(\mu+1 / 2)}, \quad 1 \leq l \leq \mu,
$$

with $C_{l}$ depending on $l$ but not $\mu$ or $r$. (The reason for the restriction $l \leq \mu$ is because we gain a factor $(\mu-l+1)(\mu-l+2) \cdots \mu r^{-l}$ from differentiating $r^{\mu} l$ times, and this would be much bigger in magnitude than $\mu^{l} r^{-l}$ for $l>>\mu$.)

For the derivatives of $K_{\mu}$, derivatives in $r$ hit either the $r^{-\mu}$ factor outside the integral in (A.2) or the $e^{-\sqrt{r^{2}+t^{2}}} / \sqrt{r^{2}+t^{2}}$ factor inside the integral. Suppose that $j$ derivatives hit the $r^{-\mu}$ factor and the remainder act inside the integral. The former gives a factor $(-\mu)(-\mu-1) \cdots(-\mu-j+1) r^{-j}$ while the latter gives terms of the form (powers of $r$ ) times $e^{-\sqrt{r^{2}+t^{2}}}$ times a sum of powers of $\left(r^{2}+t^{2}\right)^{-1 / 2}$, where the powers range from $l-j+1$ to $2(l-j)+1$. Due to the factor $t^{2 \mu}$, we can differentiate inside the integral up to $\mu$ times, and estimating $e^{-\sqrt{r^{2}+t^{2}}}\left(r^{2}+\right.$ $\left.t^{2}\right)^{-k / 2} \leq e^{-t / 2} e^{-r / 2} t^{-k}$ we end up showing

$$
\left|\left(\frac{d}{d r}\right)^{l} K_{\mu}(r)\right| \leq C_{l} \mu^{l} \frac{2^{\mu} \Gamma(2 \mu) r^{-\mu-l} e^{-r / 2}}{\Gamma(\mu+1 / 2)}, \quad 1 \leq l \leq \mu .
$$

Then, using the identity

$$
\Gamma(2 \mu)=\frac{2^{2 \mu-1}}{\sqrt{\pi}} \Gamma(\mu) \Gamma(\mu+1 / 2)
$$

in (A.3) and (A.5) we obtain (4.13) in Proposition 4.3.

To prove the properties of $a_{n, \mu}$ and $b_{n, \mu}$ in (4.14) and (4.15), we observe that such expansions are well known properties of modified Bessel functions; see 9.6.10 and 9.6.11 in [1]. The leading coefficients are given by

$$
a_{0, \mu}=\frac{\Gamma(\mu+1 / 2)}{\Gamma(\mu+1)}, \quad b_{0, \mu}=2^{-2 \mu-1}
$$

and they satisfy a recurrence relation

$$
a_{n, \mu}=\frac{a_{n-1, \mu}}{4\left(n^{2}+\mu n\right)}, \quad b_{n, \mu}=\frac{b_{n-1, \mu}}{4\left(n^{2}-\mu n\right)}, \quad 1 \leq n<\mu,
$$

which follows from substituting these expansions into the Bessel ODE. The bounds claimed in Proposition 4.3 follow directly.

To prove the bound on the error term for $I_{\mu}$, we write the function $e^{-r t}$ as its Taylor polynomial of degree $2(N-1)$ centred at $r t=0$ and use the Lagrange form 
of the remainder:

$$
e^{-r t}=\sum_{j=0}^{2 N-1} \frac{(-r t)^{j}}{j !}+(-r t)^{2 N} \frac{e^{-r^{\prime} t}}{(2 N) !}, \quad r^{\prime} \leq r .
$$

Substituting this into (A.1), we see that the Taylor polynomial yields the expansion to order $r^{\mu+2 N-2}$ while we estimate the remainder as above, showing that the $O\left(r^{2 N}\right)$ term in (4.14) is uniform in $\mu$. A similar argument shows the $O\left(r^{2 N}\right)$ term in (4.15) is uniform in $\mu$. This completes the proof of Proposition 4.3.

Proof of Lemma 4.4. Note that for any $\mu_{j} \geq 2 M$, we have

$$
\mu_{j}-M=M+\left(\mu_{j}-2 M\right) \geq M+\left(\frac{\mu_{j}-2 M}{2}\right)=\frac{\mu_{j}}{2} .
$$

Therefore,

$$
\sum_{\mu_{j} \geq 2 M} \mu_{j}^{N} \alpha^{\mu_{j}-M} \leq \sum_{\mu_{j} \geq 2 M} \mu_{j}^{N} \alpha^{\mu_{j} / 2} \leq \sum_{\mu_{j} \geq 2 M} \mu_{j}^{N} \beta^{\mu_{j} / 2} .
$$

There is an integer $N_{1}(\beta, N)>2 M$ such that for all $j \geq N_{1}(\beta, N), j^{N} \leq \beta^{-j / 4}$. It follows that

$$
\begin{aligned}
\sum_{\mu_{j} \geq 2 M} \mu_{j}^{N} \beta^{\mu_{j} / 2} & \leq \sum_{2 M \leq \mu_{j}<N_{1}(\beta, N)} \mu_{j}^{N} \beta^{\mu_{j} / 2}+\sum_{\mu_{j} \geq N_{1}(\beta, N)} \beta^{-\mu_{j} / 4} \beta^{\mu_{j} / 2} \\
& \leq\left|\left\{j: \mu_{j}<N_{1}(\beta, N)\right\}\right| N_{1}(\beta, N)^{N}+\sum_{\mu_{j} \geq N_{1}(\beta, N)} \beta^{\mu_{j} / 4} .
\end{aligned}
$$

Using Weyl's law for the eigenvalue counting function for $\Delta_{Y}$, we see that

$$
\left|\left\{j: \mu_{j} \leq \mu\right\}\right| \leq C \mu^{d-1} .
$$

Letting $\mu=N_{1}(\beta, N)$, we see that the first term in the last line of (A.7) is bounded by $C N_{1}(\beta, N)^{d+N-1}$.

Consider the second term. An implication of (A.8) is that we have

$$
\mu_{j} \geq\left(\frac{j}{C}\right)^{1 /(d-1)}
$$

for any $j \in \mathbb{N}$. Therefore,

$$
\sum_{\mu_{j} \geq N_{1}(\beta, N)} \beta^{\mu_{j} / 4} \leq \sum_{\mu_{j} \geq N_{1}(\beta, N)} \beta^{\frac{1}{4}(j / C)^{1 /(d-1)}} \leq \sum_{j \geq 0} \beta^{\frac{1}{4}(j / C)^{1 /(d-1)}} .
$$

There is $N_{2}(\beta, C) \in \mathbb{N}$ such that for all $j \geq N_{2}(\beta, C)$, we have $\frac{1}{4}(j / C)^{1 /(d-1)}>$ $\log _{\gamma} j$, where $\gamma=\beta^{-1 / 2}>1$. Then

$$
\begin{aligned}
\sum_{j \geq 1} \beta^{\frac{1}{4}(j / C)^{1 /(d-1)}} & \leq \sum_{0 \leq j<N_{2}(\beta, C)} \beta^{\frac{1}{4}(j / C)^{1 /(d-1)}}+\sum_{j \geq N_{2}(\beta, C)} \beta^{\log _{\gamma} j} \\
& \leq N_{2}(\beta, C)+\sum_{j \geq N_{2}(\beta, C)} j^{-2} \leq N_{2}(\beta, C)+\frac{\pi^{2}}{6} .
\end{aligned}
$$


The remaining part of the summation is from $M$ to $2 M$,

$\sum_{M \leq \mu_{j}<2 M} \mu_{j}^{N} \alpha^{\mu_{j}-M} \leq\left|\left\{j: \mu_{j}<2 M\right\}\right|(2 M)^{N} \leq C(2 M)^{d-1}(2 M)^{N}=C(2 M)^{d+N-1}$.

Bringing all the parts together, we have

(A.10) $\sum_{\mu_{j} \geq M} \mu_{j}^{N} \alpha^{\mu_{j}-M} \leq C(2 M)^{d+N-1}+C N_{1}(\beta, N)^{d+N-1}+N_{2}(\beta, C)+\frac{\pi^{2}}{6}<\infty$.

Note the finite constant depends on $M, N, C$, and $\beta$, but not $\alpha$, therefore we have uniform boundedness in $\alpha$.

\section{References}

[1] Abramowitz, M. and Stegun I. A.: Handbook of mathematical functions: with formulas, graphs, and mathematical tables. National Bureau of Standards Applied Mathematics Series 55, US Goverment Printing Office, Washington, D.C., 1964.

[2] Alexopoulos, G.: An application of homogenization theory to harmonic analysis: Harnack inequalities and Riesz transforms on Lie groups of polynomial growth. Canad. J. Math. 44 (1992), no. 4, 691-727.

[3] Alexopoulos, G.: Centered densities on Lie groups of polynomial volume growth. Probab. Theory Related Fields 124 (2002), no. 1, 112-150.

[4] AssaAd, J.: Riesz transforms associated to Schrödinger operators with negative potentials. Publ. Mat. 55 (2011), no. 1, 123-150.

[5] Assaad, J. And Ouhabaz, E.: Riesz transforms of Schrödinger operators on manifolds. J. Geom. Anal. 22 (2012), no. 4, 1108-1136.

[6] Auscher, P. And Ben Ali, B.: Maximal inequalities and Riesz transform estimates on $L^{p}$ spaces for Schrödinger operators with nonnegative potentials. Ann. Inst. Fourier (Grenoble) $5 \mathbf{7}$ (2007), no. 6, 1975-2013.

[7] Auscher, P., Coulhon, T., Duong, X. and Hofmann, S.: Riesz transform on manifolds and heat kernel regularity. Ann. Sci. École Norm. Sup. (4) 37 (2004), no. 6, 911-957.

[8] Auscher, P. And Coulhon, T.: Riesz transform on manifolds and Poincaré inequalities. Ann. Sc. Norm. Super. Pisa Cl. Sci. (5) 4 (2005), no. 3, 531-555.

[9] Blank, A. And Keller, J. B.: Diffraction and reflection of pulses by wedges and corners. Comm. Pure Appl. Math. 4 (1951), 75-94.

[10] Blunck, S. And Kunstmann, P. C.: Weak type $(p, p)$ estimates for Riesz transforms. Math. Z. 247 (2004), no. 1, 137-148.

[11] Brüning, J. ANd Seeley, R.: The resolvent expansion for second order regular singular operators. J. Funct. Anal. 73 (1987), no. 2, 369-429.

[12] Burq, N., Planchon, F., Stalker, J. G. And Tahvildar-Zadeh, A. S.: Strichartz estimates for the wave and Schrödinger equations with the inverse-square potential. J. Funct. Anal. 203 (2003), no. 2, 519-549.

[13] Carron, G.: Le saut en zéro de la fonction de décalage spectral. J. Funct. Anal. 212 (2004), no. 1, 222-260. 
[14] Carron, G., Coulhon, T. And Hassell, A.: Riesz transform and $L^{p}$-cohomology for manifolds with Euclidean ends. Duke Math. J. 133 (2006), no. 1, 59-93.

[15] Cheeger, J. And Taylor, M.: On the diffraction of waves by conical singularities I. Comm. Pure Appl. Math. 35 (1982), no. 3, 275-331.

[16] Cheeger, J. and Taylor, M.: On the diffraction of waves by conical singularities II. Comm. Pure Appl. Math. 35 (1982), no. 4, 487-529.

[17] Cheeger, J.: On the spectral geometry of spaces with cone-like singularities. Proc. Nat. Acad. Sci. USA 76 (1979), no. 5, 2103-2106.

[18] Coulhon, T. And Duong, X. T.: Riesz transforms for $1 \leq p \leq 2$. Trans. Amer. Math. Soc. 351 (1999), no. 3, 1151-1169.

[19] Coulhon, T. And Duong, X. T.: Riesz transform and related inequalities on noncompact Riemannian manifolds. Comm. Pure Appl. Math. 56 (2003), no. 12, $1728-1751$.

[20] Coulhon, T. And Dungey, N.: Riesz transform and perturbation. J. Geom. Anal. 17 (2007), no. 2, 213-226.

[21] ter Elst, A. F. M., Robinson, D. And Sikora, A.: Heat kernels and Riesz transforms on nilpotent Lie groups. Colloq. Math. 74 (1997), no. 2, 191-218.

[22] ter Elst, A.F. M., Robinson, D. And Sikora, A.: On second-order periodic elliptic operators in divergence form. Math. Z. 238 (2001), no. 3, 569-637.

[23] Friedlander, F. G.: The diffraction of sound pulses. I. Diffraction by a semi-infinite plane. Proc. Roy. Soc. London. Ser. A. 186 (1946), 322-344.

[24] Friedlander, F. G.: The diffraction of sound pulses. II. Diffraction by an infinite wedge. Proc. Roy. Soc. London. Ser. A. 186 (1946), 344-351.

[25] Grafakos, L.: Modern Fourier analysis. Graduate Texts in Mathematics 250, Springer, New York, 2009.

[26] Grieser, D.: Basics of the b-calculus. In Approaches to singular analysis (Berlin, 1999), 30-84. Oper. Theory Adv. Appl. 125, Birkhäuser, Basel, 2001.

[27] Guillarmou, C. And Hassell, A.: Resolvent at low energy and Riesz transform for Schrödinger operators on asymptotically conic manifolds. I. Math. Ann. 341 (2008), no. 4, 859-896.

[28] Guillarmou, C. and Hassell, A.: Resolvent at low energy and Riesz transform for Schrödinger operators on asymptotically conic manifolds. II. Ann. Inst. Fourier (Grenoble) 59 (2009), no. 4, 1553-1610.

[29] Guillarmou, C., Hassell, A. and Sikora, A.: Resolvent at low energy III: the spectral measure. Trans. Amer. Math. Soc. 365 (2013), no. 11, 6103-6148.

[30] Hassell, A. And Sikora, A.: Riesz transforms in one dimension. Indiana Univ. Math. J. 58 (2009), no. 2, 823-852.

[31] Hörmander, L.: The spectral function of an elliptic operator. Acta Math. 121 (1968), 193-218

[32] Hörmander, L.: The analysis of linear partial differential operators. III. Pseudodifferential operators. Grundlehren der Mathematischen Wissenschaften 274, SpringerVerlag, Berlin, 1985.

[33] LI, H. Q.: La transformation de Riesz sur les variétés coniques. J. Funct. Anal. 168 (1999), no. 1, 145-238.

[34] Lin, P.: The boundedness of the Riesz transform on a metric cone. Thesis, ANU, 2012; https://digitalcollections.anu.edu.au/handle/1885/9975. 
[35] Mooers, E.: Heat kernel asymptotics on manifolds with conic singularities. J. Anal. Math. 78 (1999), 1-36.

[36] Melrose, R. And Wunsch, J.: Propagation of singularities for the wave equation on conic manifolds. Invent. Math. 156 (2004), no. 2, 235-299.

[37] Melrose, R.: The Atiyah-Patodi-Singer index theorem. Research Notes in Mathematics 4, Wellesley, MA, 1993.

[38] Melrose, R.: Spectral and scattering theory for the Laplacian on asymptotically Euclidian spaces. In Spectral and scattering theory (Sanda, 1992), 85-130. Lecture Notes in Pure and Appl. Math. 161, Dekker, New York, 1994.

[39] Melrose, R.: Geometric scattering theory. Standford Lectures, Cambridge University Press, Cambridge, 1995.

[40] Riesz, M.: Sur les fonctions conjuguées. Math. Z. 27 (1927), no. 1, 218-244.

[41] SAloff-Coste, L.: Analyse sur les groupes de Lie à croissance polynomiale. Ark. Mat. 28 (1990), no. 2, 315-331.

[42] Shen, Z. W.: $L^{p}$ estimates for Schrödinger operators with certain potentials. Ann. Inst. Fourier (Grenoble) $\mathbf{4 5}$ (1995), no. 2, 513-546.

[43] Stein, E.: Harmonic analysis: real-variable methods, orthogonality, and oscillatory integrals. Princeton Mathematical Series 43, Monographs in Harmonic Analysis III, Princeton University Press, Princeton, NJ, 1993.

[44] Strichartz, R. S.: Analysis of the Laplacian on the complete Riemannian manifold. J. Funct. Anal. 52 (1983), no. 1, 48-79.

[45] Sommerfeld, A.: Mathematische Theorie der Diffraction. Math. Ann. 47 (1896), no. $2-3,317-374$.

[46] Wang, X.P.: Asymptotic expansion in time of the Schrödinger group on conical manifolds. Ann. Inst. Fourier (Grenoble) 56 (2006), no. 6, 1903-1945.

Received June 18, 2012; revised May 20, 2013.

Andrew Hassell: Department of Mathematics, Australian National University, Canberra ACT 0200, Australia.

E-mail: Andrew.Hassell@anu.edu.au

PeiJie Lin: Department of Mathematics, Australian National University, Canberra ACT 0200, Australia.

E-mail: Paul.Lin@anu.edu.au 